\title{
The Impact of DSM-IV Mental Disorders on Adherence to Combination Antiretroviral Therapy Among Adult Persons Living with HIV/AIDS: A Systematic Review
}

\author{
Sandra A. Springer • Azem Dushaj • \\ Marwan M. Azar
}

Published online: 30 May 2012

(C) The Author(s) 2012. This article is published with open access at Springerlink.com

\begin{abstract}
This is a systematic review of eighty-two published studies investigating the impact of DSM-IV mental disorders on combination antiretroviral therapy (cART) adherence and persistence among persons living with HIV/AIDS (PLWHA). Sixty-two articles examined depression, with $58 \%(N=32 / 62)$ finding lower cART adherence and persistence. Seventeen articles examined one or more anxiety disorders, with the majority finding no association with cART adherence or persistence. Eighty percent of the studies that evaluated the impact of psychotic $(N=3)$, bipolar $(N=5)$ and personality disorders $(N=2)$ on cART adherence and persistence also found no association. Seven out of the nine studies (78 \%) evaluating the impact of antidepressant treatment (ADT) on cART adherence found improvement. Adherence and depression measurements varied significantly in studies; common research measurements would improve data harmonization. More research specifically addressing the impact of other mental disorders besides depression on cART adherence and RCTs evaluating ADT on cART adherence are also needed.
\end{abstract}

Keywords HIV/AIDS - Mental illness · Adherence · Antiretroviral therapy - Systematic review · Persistence . cART $\cdot$ Depression · Anxiety · Psychotic disorders

Electronic supplementary material The online version of this article (doi:10.1007/s10461-012-0212-3) contains supplementary material, which is available to authorized users.

S. A. Springer $(\varangle) \cdot$ A. Dushaj · M. M. Azar

Yale AIDS Program, Yale University School of Medicine,

135 College Street, Suite 323, New Haven, CT 06511, USA

e-mail: Sandra.springer@yale.edu

\section{Introduction}

Combination antiretroviral therapy (cART) has greatly improved the morbidity, and decreased the mortality associated with HIV infection [1-3]. The benefits of cART, however, are typically contingent upon excellent cART adherence and persistence in order to achieve suppression of HIV-1 RNA levels and an increase in CD4 T cell lymphocytes $[4,5]$. Suboptimal adherence to cART is strongly related to viral proliferation [6, 7], drug resistance [7, 8], disease progression [9], and death [3]. Factors that can impair adherence to cART include drug addiction [10-12], alcohol use disorders [11, 13], low socioeconomic status [14], social stigma [15-17], neurocognitive disorders [18], and mental disorders [19]. When combined, mental disorders and substance use disorders among persons living with HIV/AIDS (PLWHA) synergistically increase mortality via impairing adherence to cART [20].

Mental disorders include a variety of psychiatric conditions and are defined by the fourth Diagnostic Manual of Mental Disorders (DSM-IV) [21] as 'a clinically significant behavioral or psychological impairment of an individual's normal cognitive, emotional, or behavioral functioning associated with present distress and caused by physiological or psychosocial factors'. Mental disorders are more common among PLWHA (63\%) as compared to the HIV-negative population $(30.5 \%)$ [19, 22]. In a recently published study, HIV-positive men were more likely to have any mood disorder [odds ratio $(\mathrm{OR})=6.10$ ], major depressive disorder/dysthymia $(\mathrm{OR}=3.77)$, any anxiety disorder $(\mathrm{OR}=4.02)$, and any personality disorder $(\mathrm{OR}=2.50)$ when compared to their HIV-negative samesex counterparts [23]. In another study an estimated $60 \%$ of PLWHA receiving care in North Carolina had co-morbid mental disorder symptoms [24]. 
The mental disorder most commonly associated with HIV infection is major depressive disorder (MDD) [25] with a prevalence ranging from $16.2 \%$ [26] to $36 \%$ [25]. This is a four- to seven-fold greater prevalence than in the general population (4.9\%) [27]. The large variation in prevalence rates of mental disorders has been partially attributed to differences in the specificity and sensitivity of the study instruments used [28]. Mental disorders have been associated with decreased adherence to cART and impaired HIV virologic control in several studies [14, 2936]. In one longitudinal study, HIV-positive mothers with co-morbid mental disorders were approximately six times more likely to die than adherent participants with no depressive symptoms [32]. A recently published metaanalysis of 95 independent studies of PLWHA with depressive disorders concluded that depression was significantly associated with nonadherence to cART ( $r=$ 0.19 ; $95 \% \mathrm{CI}=0.14-0.25, p<0.0001)$ [37]. This metaanalysis, however, was not a systematic review of the studies of depression and adherence to cART. To the authors' knowledge there has not yet been a systematic review of studies published in the English literature evaluating all major Axis I and II DSM-IV mental disorders on cART adherence and persistence among adult PLWHA.

The specific aim of this paper is to therefore systematically review studies evaluating the impact of all of major Axis I and II DSM-IV mental disorders, excluding substance use disorders, on combination antiretroviral adherence and persistence among PLWHA. Though major depression is the principal focus of this review due to its higher prevalence, other mental disorders such as anxiety and psychotic disorders will also be examined as they have also been reported to negatively impact participant adherence [34, 38].

\section{Methods}

\section{Data Search}

PubMed, Scopus and Web of Knowledge were queried for peer-reviewed original human research papers published in English from 1996 to December 2011. Google Scholar was also reviewed for details, full text, and additional articles. The search for this systematic review took place from November 2010 to December 2011. Keywords and their combinations used in the search are available in an on-line appendix. Papers were included if they were conducted in other countries outside of the United States, given the high prevalence of mental disorders among PLWHA throughout the world.
Study Selection and Inclusion/Exclusion Criteria

Figure 1 represents a PRISMA (Preferred Reporting Items for Systematic reviews and Meta-Analyses) flow diagram for this systematic review [39]. The original search resulted in 4,302 total documents (PubMed-2,151 articles, Scopus-1,847 articles, Web of Knowledge-304 articles) of which 2,676 articles remained after eliminating duplicate articles. Of these, 659 articles met the following inclusion criteria for the primary selection: [1] mental disorders, including psychotic, mood, anxiety, somatoform, and personality disorders as defined by International Classification of Diseases (ICD-9 and ICD-10) [40, 41], Diagnostic and Statistical Manual of Mental Disorders (DSM-IV Axis I or II diagnosis) [21] or mental health symptoms (many studies used screening tools for mental disorder symptoms, rather than specific mental disorder diagnostic measures) [42]); and [2] antiretroviral treatment adherence or persistence. Articles included in this systematic review evaluated the impact of DSM-IV mental disorders or mental health symptoms on at least one of the following cART medication terms: (a) Adherence (synonym: compliance), defined as "the extent to which a participant acts in accordance with the prescribed interval, and dose of a dosing regimen" or "percentage of correctly timed doses (doses taken/doses prescribed $\times 100$ )" [43] and (b) persistence, defined as "the duration of time of initiation to discontinuation of therapy (by the participant)" [43].

One reviewer $(A D)$ identified the papers included in the tables provided within this manuscript and in the electronic appendix. A second independent reviewer (MA) applied selection criteria to a random sample consisting of $20 \%$ of articles from the primary selection. Agreement between reviewers was assessed using Cohen's kappa $(\kappa)$ statistic and was equal to 0.69 [44]. Where there were differences between the two reviewers, a third reviewer (SAS) made the final decision as to whether the article should be included or excluded from the systematic review. Subsequently, 82 studies met the final inclusion criteria for this systematic review.

\section{Data Extraction}

Standardized data collection forms were used to extract all data including: study authors; study site; year and duration of study; study design; population characteristics; sample size; mental disorder studied and the tool used to screen and diagnose the mental disorder; definition of and the tool used to measure cART adherence. In cases where no standardized tool was used for the mental disorder or cART adherence, the tools used in the studies were noted in the data collection form. The data collection form was similar to Table 1 and the data was extracted at least twice from 


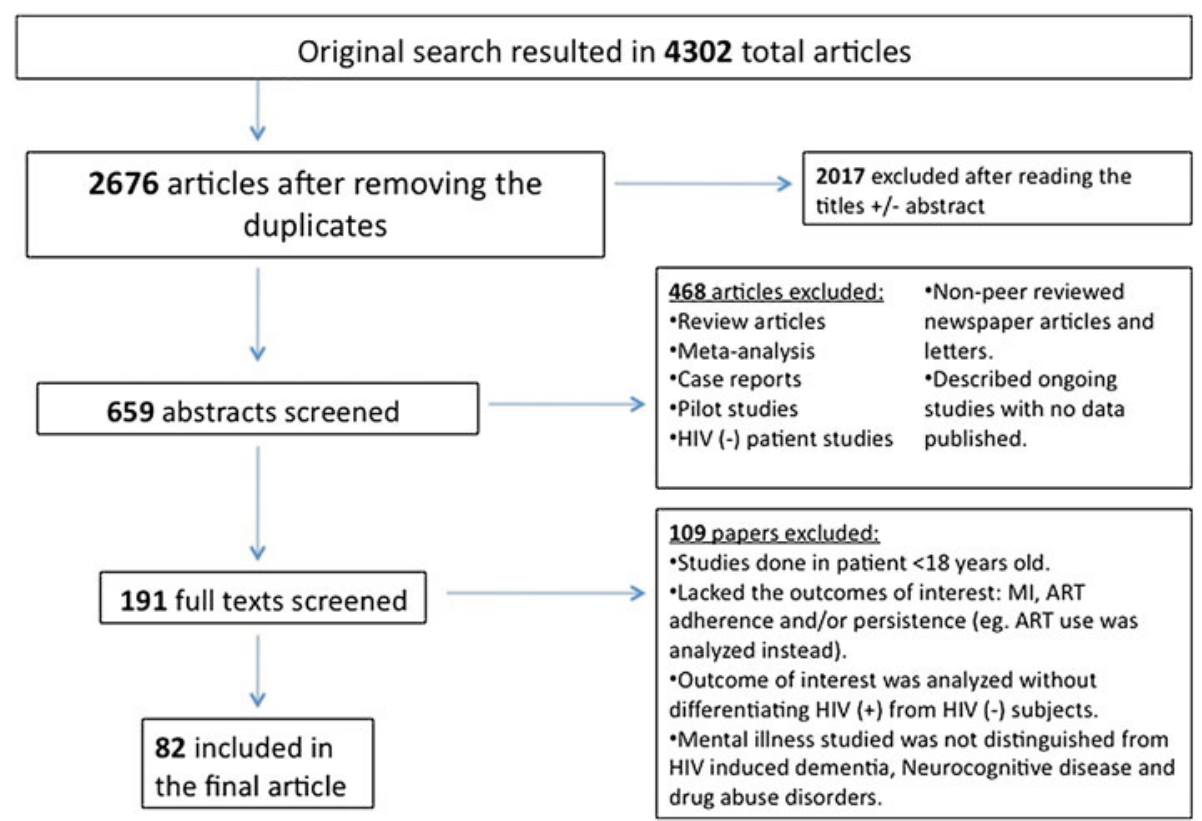

Fig. 1 Systematic review study selection flow diagram

each article by one single author (AD). The study characteristics in the tables were evaluated by one author (AD). If inconsistencies in definitions of adherence or mental disorder were identified then further appraisal of the particular issue would be carried out by two authors (AD and MZ) and noted in the tables. For example, if it was identified that the study did not use a standardized tool to create a definition of depression, e.g. 'depression defined as ever being seen by a psychiatrist or taking ADT', then the problem with the particular definition would be written in the extraction table, e.g. 'definition of depression was misleading because it may have included persons with a past medical history of depression who are no longer depressed introducing bias into results'.

In order to clearly present the results, the data were separated into four categories according to the mental disorder that was the main focus of the studies. Table 1 summarizes studies that evaluated 'the impact of depressive disorders on cART adherence and persistence'. These constituted the majority of articles reviewed $(62 / 82 ; 76 \%)$. Tables 2, 3 and 4 are presented as an electronic appendix. Table 2 summarizes studies that examined the impact of specific mental disorders other than depression on cART adherence and persistence'. Table 3 presents studies that evaluated the 'impact of mental disorders not specified per DSM-IV diagnostic criteria on cART adherence and persistence'. Finally, Table 4 summarizes articles that evaluated 'the impact of pharmacologic antidepressant treatment (ADT) on cART adherence' among PLWHA with comorbid depressive disorders. Due to the length and scope of the manuscript, behavioral treatments of depressive disorders were not included in this systematic review. In cases when cART persistence was measured instead of cART adherence, the term used by the authors was noted in the tables.

\section{Results}

This systematic review examines 82 studies evaluating the impact of mental disorders and mental disorder symptoms (including mood, anxiety, psychotic, and personality disorders) on two primary outcomes: adherence to, and persistence on cART (see Tables 1 in the manuscript and the Tables 2, 3 and 4 within the electronic appendix).

Antiretroviral Adherence Measurements

Adherence to cART was assessed using multiple methods (as highlighted in the Tables). Seventy-seven of the articles used only one measurement of adherence, four studies used two adherence measurements and one used three different measures of cART adherence. These included: self-reports (participants recall) $(N=65)$, electronic drug monitors (EDMs) $(N=9)$, pharmacy refill record review $(N=6)$, hospital or clinic record review $(N=2)$, unannounced pill counts $(N=5)$, and directly observed therapy (DOT) $(N=1)$, and some of the studies used a combination of the aforementioned measures of adherence to cART $(N=5)$.

The time period over which adherence was assessed ranged from 1 day to 1 year prior to the interview. Among the self-report instruments, adherence recall periods ranged 
Table 1 Impact of depressive disorders on cART adherence: study characteristics

\begin{tabular}{lllll}
\hline $\begin{array}{l}\text { Author, } \\
\text { publication year, } \\
\text { location }\end{array}$ & $\begin{array}{l}\text { Study design and } \\
\text { evaluation period }\end{array}$ & $\begin{array}{l}\text { PLWHA } \\
\text { population, } \\
\text { sample size }\end{array}$ & $\begin{array}{l}\text { Adherence: } \\
\text { measurement (M), } \\
\text { definition (D) and time } \\
\text { period (T) }\end{array}$ & $\begin{array}{l}\text { Depressive disorder and } \\
\text { scale used to measure } \\
\text { depressive disorder }\end{array}$ \\
\end{tabular}

\section{Arnsten, J. H., X. Cross-sectional \\ Li, et al. (2007) study (2001-2005) \\ USA \\ Boarts, J. M., E. Prospective cohort M. Sledjeski, study (3-month et al. (2006) follow-up) \\ USA}

I. Depressive disorders associated with decreased adherence to cART

Buathong, N., N. Hiransuthikula, et al. (2009)

Cross-sectional

Thailand

Carrieri, M. P.,

Cohort study (Oct

M. A. Chesney,

et al. (2003)

France study (Oct 2007Jan 2008)

\author{
636 adults \\ reporting \\ recent IDU and \\ sexual activity \\ with an \\ opposite- \\ gender partner
}

57 participants

\section{M: Self-report (AACTG adapted questionnaire) \\ D: Continuous variable}

T: Previous week

379 participants
on cART
recruited from
the
immunology
and sexual
transmitted
disease clinics
96 IDU
participants
(initially
adherent to
cART)

18 months on cART)
M: Self-report

D: $\geq 95 \%$ adherence

T: Previous 1 month

\section{M: Self-report}

D: Adherence failure: $<80 \%$ adherence or did not "totally"
Carrieri, M. P., C. Cohort study (May Leport, et al. (2006) France
1997-Jun 1999;

5-year follow-up)

Cruess, D. G., S. Prospective cohort C. Kalichman, study (Mar 2005et al. (2011) Oct 2008) follow their prescribed regimen

T: Previous week or at any visit before the 18th month of treatment

970 participants

M: Self-report (ACTG questionnaire)

D: High (100\% adherence), Moderate (80-99.9\% adherence) and Poor $(<80 \%$ adherence)

T: Previous 4 days

324 participants M: Unannounced pill count

D: Continuous variable (percentage adherence)

T: Previous 3 months
Depression-CES-D

Depression-BDI-II

Depression-7-item depression component of the BSI$$
\text { Depression-BDI-II }
$$

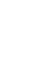

Multivariate analysis (MVA): Depression was significantly associated with poorer adherence, $(\mathrm{OR}=0.74,95 \%$ $\mathrm{CI}=0.58-0.94$, $p<0.05)$

MVA: Depressive symptoms at baseline predicted lower levels of adherence at followup $\left(R^{2}=0.076\right.$, $p \leq 0.05)$

MVA: Depression was significantly associated with non-adherence $(\mathrm{AOR}=4.68 ; 95 \%$ $\mathrm{CI}=2.77-7.88$, $p<0.001$ )

Depression-CES-D

Depression-CES-D (score $>16$ )

Depression-CES-D
MVA: Depression was significantly related to adherence failure $(\mathrm{OR}=2.5,95 \%$ $\mathrm{CI}=1.0-6.0$, $p=0.044$ )
MVA: Depression was independently associated with moderate or poor adherence but not with high adherence (Coeff $=0.18,95 \%$ $\mathrm{CI}=0.07-0.29$ )

MVA: Higher mean depression score was significantly associated with lower antiretroviral medication adherence $(\beta(4,300)=-0.22$, $p<0.001)$ 
Table 1 continued

\begin{tabular}{|c|c|c|c|c|c|}
\hline $\begin{array}{l}\text { Author, } \\
\text { publication year, } \\
\text { location }\end{array}$ & $\begin{array}{l}\text { Study design and } \\
\text { evaluation period }\end{array}$ & $\begin{array}{l}\text { PLWHA } \\
\text { population, } \\
\text { sample size }\end{array}$ & $\begin{array}{l}\text { Adherence: } \\
\text { measurement (M), } \\
\text { definition (D) and time } \\
\text { period (T) }\end{array}$ & $\begin{array}{l}\text { Depressive disorder and } \\
\text { scale used to measure } \\
\text { depressive disorder }\end{array}$ & $\begin{array}{l}\text { Impact of depressive } \\
\text { disorder on adherence }\end{array}$ \\
\hline $\begin{array}{l}\text { Diiorio, C., F. } \\
\text { McCarty, et al. } \\
\text { (2009) USA }\end{array}$ & $\begin{array}{l}\text { Cross-sectional } \\
\text { study }\end{array}$ & 236 participants & $\begin{array}{l}\text { M: UCSF Adherence } \\
\text { Questionnaire } \\
\text { D: Reasons for missing } \\
\text { medication } \\
\text { T: Previous } 30 \text { days }\end{array}$ & Depression-CES-D & $\begin{array}{l}\text { MVA: Significant } \\
\text { negative association } \\
\text { was found between } \\
\text { depression and poor } \\
\text { adherence (fewer } \\
\text { depressive symptoms } \\
\text { supported greater } \\
\text { adherence) }\end{array}$ \\
\hline $\begin{array}{l}\text { Do, N. T., K. } \\
\text { Phiri, et al. } \\
\text { (2010) } \\
\text { Botswana } \\
\text { (Afrika) }\end{array}$ & $\begin{array}{l}\text { Cross-sectional } \\
\text { study (Apr-May } \\
\text { 2005) }\end{array}$ & 300 participants & $\begin{array}{l}\text { M: Self-report, } \\
\text { institutional adherence } \\
\text { (pharmacy refill rate), } \\
\text { and a culturally } \\
\text { modified Morisky scale } \\
\text { D: [1] } 100 \% \text { adherence } \\
\text { in the past } 4 \text { days, [2] } \\
100 \% \text { adherence in the } \\
\text { past } 1 \text { month, and [3] } \\
100 \% \text { refill in the past } \\
3 \text { months } \\
\text { T: Previous } 4 \text { days, } \\
1 \text { month and prior } \\
\text { consecutive } 3 \text { months }\end{array}$ & Depression-BDI & $\begin{array}{l}\text { MVA: Depression was a } \\
\text { significant predictor of } \\
\text { non-adherence } \\
(p<0.02)\end{array}$ \\
\hline $\begin{array}{l}\text { Etienne, M., M. } \\
\text { Hossain, et al. } \\
\text { (2010) Kenya, } \\
\text { Uganda, } \\
\text { Zambia, } \\
\text { Nigeria, } \\
\text { Rwanda }\end{array}$ & $\begin{array}{l}\text { Cross-sectional } \\
\text { study } \\
\text { (Aug 2004-Apr } \\
\text { 2005) }\end{array}$ & 921 participants & $\begin{array}{l}\text { M: Self-report } \\
\text { D: Non-adherence: [1] } \\
\text { Missing } 1 \text { or more } \\
\text { doses; [2] missing } 1 \text { or } \\
\text { more appointments } \\
\text { T: Previous week (for } \\
\text { doses); previous } \\
3 \text { months (for } \\
\text { appointments) }\end{array}$ & $\begin{array}{l}\text { Depression-Factor } \\
\text { scores (low, medium, } \\
\text { high) constructed from } \\
\text { three questions }\end{array}$ & $\begin{array}{l}\text { MVA: Only high } \\
\text { depression score was } \\
\text { associated with non- } \\
\text { adherence }(\mathrm{OR}=0.57 \text {, } \\
95 \% \mathrm{CI}=0.39-0.84 \text {, } \\
p<0.01)\end{array}$ \\
\hline $\begin{array}{l}\text { Gonzalez, J. S., } \\
\text { C. Psaros, et al. } \\
\text { (2011) USA }\end{array}$ & Cohort study & $\begin{array}{l}91 \text { participants } \\
\text { in methadone } \\
\text { maintenance }\end{array}$ & $\begin{array}{l}\text { M: EDMs } \\
\text { D: } 100 \% \text { adherence (no } \\
\text { missed doses) } \\
\text { T: Previous } 2 \text { weeks }\end{array}$ & $\begin{array}{l}\text { Depression: clinician } \\
\text { ratings (MINI, Clinical } \\
\text { Global Impression } \\
\text { Scale and MADRS) } \\
\text { and self-report (BDI- } \\
\text { SF) }\end{array}$ & $\begin{array}{l}\text { MVA: Each unit increase } \\
\text { in the Clinical Global } \\
\text { Impression Scale was } \\
\text { associated with } 75 \% \\
\text { increased odds of non- } \\
\text { adherence (OR }=1.75 \text {, } \\
p=0.002,95 \% \\
\text { CI }=1.23-2.48) \text {. For } \\
\text { each SD MADRS } \\
\text { increase, there was a } \\
2.6 \text {-fold increased odds } \\
\text { of non-adherence } \\
(\text { OR }=2.60, \\
p=0.001,95 \% \\
\text { CI }=1.45-4.67)\end{array}$ \\
\hline $\begin{array}{l}\text { Kacanek, D., D. } \\
\text { L. Jacobson, } \\
\text { et al. (2010) } \\
\text { USA }\end{array}$ & $\begin{array}{l}\text { Longitudinal cohort } \\
\text { study (Feb 1995- } \\
\text { Dec/2004) }\end{array}$ & 225 participants & $\begin{array}{l}\text { M: Self-report } \\
\text { D: Suboptimal } \\
\text { adherence: missing } \\
>5 \% \text { of cART doses } \\
\text { T: Previous } 7 \text { days }\end{array}$ & $\begin{array}{l}\text { Incident depression- } \\
\text { Burnam's interviewer } \\
\text { administered 8-item } \\
\text { screening tool }\end{array}$ & $\begin{array}{l}\text { MVA: Subjects who } \\
\text { developed depressive } \\
\text { symptoms had a } \\
\text { twofold greater risk of } \\
\text { suboptimal adherence } \\
\text { at follow up } \\
\text { (RR }=1.8,95 \% \\
\text { CI }=1.1-3.0)\end{array}$ \\
\hline
\end{tabular}


Table 1 continued

\begin{tabular}{|c|c|c|c|c|c|}
\hline $\begin{array}{l}\text { Author, } \\
\text { publication year, } \\
\text { location }\end{array}$ & $\begin{array}{l}\text { Study design and } \\
\text { evaluation period }\end{array}$ & $\begin{array}{l}\text { PLWHA } \\
\text { population, } \\
\text { sample size }\end{array}$ & $\begin{array}{l}\text { Adherence: } \\
\text { measurement }(\mathrm{M}) \text {, } \\
\text { definition }(\mathrm{D}) \text { and time } \\
\text { period }(\mathrm{T})\end{array}$ & $\begin{array}{l}\text { Depressive disorder and } \\
\text { scale used to measure } \\
\text { depressive disorder }\end{array}$ & $\begin{array}{l}\text { Impact of depressive } \\
\text { disorder on adherence }\end{array}$ \\
\hline $\begin{array}{l}\text { Kleeberger, C. } \\
\text { A., J. Buechner, } \\
\text { et al. (2004) } \\
\text { USA }\end{array}$ & $\begin{array}{l}\text { Longitudinal cohort } \\
\text { study (Oct 1998- } \\
\text { Oct 2000) }\end{array}$ & 597 men & $\begin{array}{l}\text { M: Self-report } \\
\text { D: Dichotomized: } 100 \% \\
\text { adherence or less than } \\
100 \% \text { adherence } \\
\text { T: Previous } 4 \text { days }\end{array}$ & Depression-CES-D & $\begin{array}{l}\text { MVA: CES-D score }>16 \\
\text { was an independent } \\
\text { predictor of decreasing } \\
\text { adherence }(\mathrm{OR}=1.8, \\
p=0.03)\end{array}$ \\
\hline $\begin{array}{l}\text { Lazo, M., S. } \\
\text { J. Gange, et al. } \\
\text { (2007) USA }\end{array}$ & $\begin{array}{l}\text { Longitudinal cohort } \\
\text { study (1999-2004) }\end{array}$ & $\begin{array}{l}1,904 \\
\text { participants } \\
(640 \text { men and } \\
1,304 \text { women })\end{array}$ & $\begin{array}{l}\text { M: Self-report (AACTG } \\
\text { questionnaire) } \\
\text { D: Dichotomized: } 100 \% \\
\text { adherence or less than } \\
100 \% \text { adherence } \\
\text { T : Previous } 4 \text { days } \\
\text { (men) or } 3 \text { days } \\
\text { (women) }\end{array}$ & $\begin{array}{l}\text { Symptoms of depression } \\
\text { were measured using } \\
\text { the CES-D }\end{array}$ & $\begin{array}{l}\text { MVA: Symptoms of } \\
\text { depression were a } \\
\text { significant independent } \\
\text { predictor of decreased } \\
\text { adherence among men } \\
(\mathrm{OR}=1.44,95 \% \\
\mathrm{CI}=1.06-1.95, \\
p<0.05), \text { but not } \\
\text { women }\end{array}$ \\
\hline $\begin{array}{l}\text { Li, L., S. J. Lee, } \\
\text { et al. (2010) } \\
\text { Thailand }\end{array}$ & $\begin{array}{l}\text { Cross-sectional } \\
\text { study } 2007\end{array}$ & 386 participants & $\begin{array}{l}\text { M: Self-report (of failing } \\
\text { to adhere to cART) } \\
\text { D: }<100 \% \text { adherence } \\
\text { T: Previous } 1 \text { month }\end{array}$ & $\begin{array}{l}\text { Depressive symptoms: } \\
\text { 15-item screening test } \\
\text { developed and used } \\
\text { previously in Thailand } \\
\text { (Thai Department of } \\
\text { Mental Health, 2006) }\end{array}$ & $\begin{array}{l}\text { MVA: Depression was a } \\
\text { significant predictor of } \\
\text { poor adherence to } \\
\text { cART }(\mathrm{OR}=0.69, \\
p=0.03)\end{array}$ \\
\hline $\begin{array}{l}\text { Olisah, V. O., O. } \\
\text { Baiyewu, et al. } \\
\text { (2010) Zaria }\end{array}$ & $\begin{array}{l}\text { Cross-sectional } \\
\text { study (Sep-Dec } \\
\text { 2006) }\end{array}$ & 310 participants & $\begin{array}{l}\text { M: Self-report } \\
\text { D: Poor adherence: } \\
\quad<100 \% \text { adherence } \\
\text { T: Previous } 7 \text { days }\end{array}$ & $\begin{array}{l}\text { Depression-CES-D and } \\
\text { SCAN to confirm } \\
\text { depression diagnosis }\end{array}$ & $\begin{array}{l}\text { Bivariate analysis } \\
\text { (BVA): cART } \\
\text { adherence in } \\
\text { participants with } \\
\text { depressive disorder } \\
(36.4 \%) \text { was } \\
\text { significantly poorer } \\
\text { than that in non- } \\
\text { depressed participants } \\
(78.9 \%), \\
\left(\chi^{2}=34.657, \text { df }=1,\right. \\
p<0.05)\end{array}$ \\
\hline $\begin{array}{l}\text { Phillips, K. D., L. } \\
\text { Moneyham, } \\
\text { et al. (2005) } \\
\text { USA }\end{array}$ & $\begin{array}{l}\text { Cross-sectional } \\
\text { study }\end{array}$ & $\begin{array}{l}173 \text { low-income, } \\
\text { women living } \\
\text { with HIV/ } \\
\text { AIDS in the } \\
\text { rural } \\
\text { southeastern } \\
\text { United States }\end{array}$ & $\begin{array}{l}\text { M: Self-report (AACTG } \\
\text { questionnaire) } \\
\text { D: Continuous variable } \\
\text { T: Previous } 1 \text { and } \\
3 \text { months }\end{array}$ & $\begin{array}{l}\text { Depression-CES-D, } \\
\text { classified as mild } \\
\text { depressive symptoms } \\
\text { (CES-D score } 8-15 \text { ) } \\
\text { and severe depressive } \\
\text { symptoms (CES-D } \\
\text { score } 16 \text { or greater) }\end{array}$ & $\begin{array}{l}\text { BVA: Severe depressive } \\
\text { symptoms were } \\
\text { significantly associated } \\
\text { with non-adherence } \\
(p=0.0002)\end{array}$ \\
\hline $\begin{array}{l}\text { Protopopescu, C., } \\
\text { F. Raffi, et al. } \\
\text { (2009) France }\end{array}$ & $\begin{array}{l}10 \text { year Cohort } \\
\text { study (enrolled } \\
\text { May 1997-June } \\
\text { 1999) }\end{array}$ & $\begin{array}{l}1,010 \\
\text { participants }\end{array}$ & $\begin{array}{l}\text { M: Self-report (AACTG } \\
\text { questionnaire) } \\
\text { D: Non-adherence: } \\
\quad<100 \% \text { adherence } \\
\text { T: Previous } 4 \text { weeks }\end{array}$ & $\begin{array}{l}\text { Depression-CES-D, } \\
\text { participants were } \\
\text { classified as having } \\
\text { depressive symptoms if } \\
\text { their CES-D score was } \\
>17 \text { for men and }>23 \\
\text { for women }\end{array}$ & $\begin{array}{l}\text { MVA: Depressive } \\
\text { symptoms were } \\
\text { independently } \\
\text { associated with non- } \\
\text { adherence } \\
\text { (Coeff }=0.171,95 \% \\
\text { CI }=0.087-0.283, \\
p<0.001)\end{array}$ \\
\hline
\end{tabular}


Table 1 continued

\begin{tabular}{|c|c|c|c|c|c|}
\hline $\begin{array}{l}\text { Author, } \\
\text { publication year, } \\
\text { location }\end{array}$ & $\begin{array}{l}\text { Study design and } \\
\text { evaluation period }\end{array}$ & $\begin{array}{l}\text { PLWHA } \\
\text { population, } \\
\text { sample size }\end{array}$ & $\begin{array}{l}\text { Adherence: } \\
\text { measurement (M), } \\
\text { definition (D) and time } \\
\text { period (T) }\end{array}$ & $\begin{array}{l}\text { Depressive disorder and } \\
\text { scale used to measure } \\
\text { depressive disorder }\end{array}$ & $\begin{array}{l}\text { Impact of depressive } \\
\text { disorder on adherence }\end{array}$ \\
\hline $\begin{array}{l}\text { Rao, D., B. } \\
\text { J. Feldman, } \\
\text { et al. (2011) } \\
\text { USA }\end{array}$ & $\begin{array}{l}\text { Cross-sectional } \\
\text { study (Feb-Nov } \\
\text { 2009) }\end{array}$ & 720 participants & $\begin{array}{l}\text { M: Self-report (AACTG } \\
\text { questionnaire) and } \\
\text { VAS } \\
\text { D: VAS adherence item } \\
\text { was converted into } \\
\text { 4-category ordinal } \\
\text { scale, perfect } \\
\text { adherence = } 100 \% \\
\text { adherence on VAS } \\
\text { T: Previous } 4 \text { days and } \\
4 \text { weeks }\end{array}$ & $\begin{array}{l}\text { Depressive symptoms: } \\
\text { PHQ-9 }\end{array}$ & $\begin{array}{l}\text { MVA: Depressive } \\
\text { symptoms had a } \\
\text { moderate negative } \\
\text { effect on HIV } \\
\text { medication adherence } \\
\text { (stand. } b=-0.17 \text {, } \\
p<0.01 \text { ) }\end{array}$ \\
\hline $\begin{array}{l}\text { Rodkjaer, L., T. } \\
\text { Laursen, et al. } \\
\text { (2010) } \\
\text { Denmark }\end{array}$ & $\begin{array}{l}\text { Cross-sectional } \\
\text { study (May 2005- } \\
\text { Sep 2005) }\end{array}$ & 205 participants & $\begin{array}{l}\text { M: Self-report (AACTG } \\
\text { questionnaire) } \\
\text { D: Non-adherence: } \\
<100 \% \text { adherence } \\
\text { T: Previous } 4 \text { days }\end{array}$ & Depression—BDI-II & $\begin{array}{l}\text { MVA: Participants at } \\
\text { risk of depression } \\
(\mathrm{BDI}>20) \text { were more } \\
\text { likely to be non- } \\
\text { adherent }(\mathrm{OR}=5.7 \text {, } \\
95 \% \mathrm{CI}=1.7-18.6)\end{array}$ \\
\hline $\begin{array}{l}\text { Rodkjaer, L., T. } \\
\text { Laursen, et al. } \\
\text { (2011) } \\
\text { Denmark }\end{array}$ & $\begin{array}{l}\text { Longitudinal cohort } \\
\text { study (May 2005- } \\
\text { Sep 2008) }\end{array}$ & $\begin{array}{l}205 \text { participants } \\
\text { at baseline (in } \\
\text { 2005) and } 148 \\
\text { participants at } \\
\text { follow-up (in } \\
\text { 2008) }\end{array}$ & $\begin{array}{l}\text { M: Self-report (AACTG } \\
\text { questionnaire) } \\
\text { D: Non-adherence: } \\
<100 \% \text { adherence (in } \\
\text { 2005) or stopping } \\
\text { cART for } 7 \text { days or } \\
\text { more during the last } \\
12 \text { months (in 2008) } \\
\text { T: Previous } 4 \text { days }\end{array}$ & Depression-BDI-II & $\begin{array}{l}\text { MVA: Participants at } \\
\text { risk of moderate to } \\
\text { major depression } \\
\text { (BDI > 20) were more } \\
\text { likely to be non- } \\
\text { adherent to cART in } \\
\text { the } 4 \text { days prior to } \\
\text { assessment in 2005, } \\
\text { and more likely to have } \\
\text { stopped cART for } \\
7 \text { days or more during } \\
\text { the last } 12 \text { months in } \\
2008\end{array}$ \\
\hline $\begin{array}{l}\text { Royal, S. W., D. } \\
\text { P. Kidder, et al. } \\
\text { (2009) USA }\end{array}$ & $\begin{array}{l}\text { Cross-sectional } \\
\text { study (July 2004- } \\
\text { May 2005) }\end{array}$ & $\begin{array}{l}644 \text { homeless or } \\
\text { unstably } \\
\text { housed } \\
\text { PLWHA }\end{array}$ & $\begin{array}{l}\text { M: Self-report } \\
\text { D: Continuous variable } \\
\text { T: Previous } 2 \text { and } 7 \text { days }\end{array}$ & Depression—CES-D & $\begin{array}{l}\text { MVA: Depression was } \\
\text { associated with } \\
\text { decreased } 2 \text { day } \\
(\mathrm{AOR}=1.73,95 \% \\
\mathrm{CI}=1.28-2.34 \\
p=0.0003) \text { and } \\
7 \text { days }(\mathrm{AOR}=1.91, \\
95 \% \mathrm{CI}=1.35-2.72 \\
p=0.0003 \text { ) adherence }\end{array}$ \\
\hline $\begin{array}{l}\text { Sarna, A., S. } \\
\text { Pujari, et al. } \\
\text { (2008) India }\end{array}$ & $\begin{array}{l}\text { Cross-sectional } \\
\text { study (May-Aug } \\
\text { 2004) }\end{array}$ & 310 participants & $\begin{array}{l}\text { M: Self-report (AACTG } \\
\text { questionnaire) } \\
\text { D: } \geq 90 \% \text { adherence } \\
\text { T: Previous } 4 \text { days }\end{array}$ & Depression-BDI II & $\begin{array}{l}\text { MVA: Severe depression } \\
(\mathrm{AOR}=4.48,95 \% \\
\mathrm{CI}=1.64-12.27 \\
p=0.003) \text { was } \\
\text { associated with lower } \\
\text { adherence }\end{array}$ \\
\hline $\begin{array}{l}\text { Schuman, P. } \\
\text { (2001) USA }\end{array}$ & $\begin{array}{l}\text { Cross-sectional } \\
\text { study (Dec 1996- } \\
\text { Dec 1997) }\end{array}$ & $\begin{array}{l}371 \text { women with } \\
\text { advanced HIV } \\
\text { disease }\end{array}$ & $\begin{array}{l}\text { M: Self-reported cART } \\
\text { adherence } \\
\text { D:Self-report of taking } \\
>75 \% \text { of their cART } \\
\text { (as frequently as } \\
\text { prescribed or almost all } \\
\text { the time) } \\
\text { T: Previous } 2 \text { weeks }\end{array}$ & $\begin{array}{l}\text { Depression-CES-D } \\
(\text { CES-D >15) }\end{array}$ & $\begin{array}{l}\text { MVA: Depressive mood } \\
\text { was significantly } \\
\text { associated with poorer } \\
\text { adherence }(\mathrm{OR}=0.34, \\
95 \% \mathrm{CI}=0.18-0.64, \\
p<0.001)\end{array}$ \\
\hline
\end{tabular}


Table 1 continued

\begin{tabular}{|c|c|c|c|c|c|}
\hline $\begin{array}{l}\text { Author, } \\
\text { publication year, } \\
\text { location }\end{array}$ & $\begin{array}{l}\text { Study design and } \\
\text { evaluation period }\end{array}$ & $\begin{array}{l}\text { PLWHA } \\
\text { population, } \\
\text { sample size }\end{array}$ & $\begin{array}{l}\text { Adherence: } \\
\text { measurement }(\mathrm{M}) \text {, } \\
\text { definition }(\mathrm{D}) \text { and time } \\
\text { period }(\mathrm{T})\end{array}$ & $\begin{array}{l}\text { Depressive disorder and } \\
\text { scale used to measure } \\
\text { depressive disorder }\end{array}$ & $\begin{array}{l}\text { Impact of depressive } \\
\text { disorder on adherence }\end{array}$ \\
\hline $\begin{array}{l}\text { Singh, N., C. } \\
\text { Squier, et al. } \\
\text { (1996) USA }\end{array}$ & $\begin{array}{l}\text { Longitudinal } \\
\text { observational } \\
\text { study }\end{array}$ & $\begin{array}{l}46 \text { VA Medical } \\
\text { Center } \\
\text { participants }\end{array}$ & $\begin{array}{l}\text { M: Computerized } \\
\text { pharmacy refill records } \\
\text { D: Non-compliant: filling } \\
\quad<80 \% \text { of medication } \\
\text { T: Previous } 1 \text { month }\end{array}$ & $\begin{array}{l}\text { Depression-BDI and } \\
\text { POMS }\end{array}$ & $\begin{array}{l}\text { MVA: POMS }>42 \text { was } \\
\text { significantly associated } \\
\text { with non-compliance } \\
(\mathrm{OR}=1.4,95 \% \\
\mathrm{CI}=1.1-1.8 \\
p<0.01) \text { but BDI } \\
\text { score was not }\end{array}$ \\
\hline $\begin{array}{l}\text { Spire, B., S. } \\
\text { Duran, et al. } \\
\text { (2002) France }\end{array}$ & $\begin{array}{l}\text { Prospective cohort } \\
\text { study with (May- } \\
\text { Oct 1997; follow- } \\
\text { up visit after } \\
4 \text { months) }\end{array}$ & $\begin{array}{l}445 \text { participants } \\
\text { who were } \\
\text { started on PI }\end{array}$ & $\begin{array}{l}\text { M: Self-report } \\
\text { D: } 100 \% \text { adherence } \\
\text { T: Previous } 4 \text { days }\end{array}$ & Depression-CES-D & $\begin{array}{l}\text { BVA: Baseline } \\
\text { depression was not } \\
\text { associated with future } \\
\text { non-adherence; but an } \\
\text { increase in CES-D } \\
\text { score during the } \\
\text { 4-months period of } \\
\text { treatment was } \\
\text { significantly associated } \\
\text { with non-adherence }\end{array}$ \\
\hline $\begin{array}{l}\text { Tadios, Y. and G. } \\
\text { Davey (2006) } \\
\text { Ethiopia }\end{array}$ & $\begin{array}{l}\text { Cross-sectional } \\
\text { study (Dec 2004- } \\
\text { Jan 2005) }\end{array}$ & $\begin{array}{l}431 \text { participants } \\
\text { on cART }\end{array}$ & $\begin{array}{l}\text { M: Self-report } \\
\text { D: } \geq 95 \% \text { adherence } \\
\text { T: Previous 1, } 3 \text { and } \\
7 \text { days }\end{array}$ & $\begin{array}{l}\text { Depression-BDI (cut- } \\
\text { off }=14) \text { (depression } \\
\text { used as dependent } \\
\text { variable) }\end{array}$ & $\begin{array}{l}\text { MVA: Adherence to } \\
\text { cART was significantly } \\
\text { associated with not } \\
\text { being depressed } \\
\text { (AOR }=2.8,95 \% \\
\text { CI }=1.5-5.4 \\
p=0.002)\end{array}$ \\
\hline $\begin{array}{l}\text { Tucker, J. S., M. } \\
\text { A. Burnam, } \\
\text { et al. (2003) } \\
\text { USA }\end{array}$ & $\begin{array}{l}\text { Cohort study (Jan } \\
\text { 1996-Jan 1998) }\end{array}$ & $\begin{array}{l}1910 \\
\text { participants }\end{array}$ & $\begin{array}{l}\text { M: Self-report to } 3 \\
\text { adherence questions } \\
\text { D: } 100 \% \text { adherence } \\
\text { T: Previous week }\end{array}$ & $\begin{array}{l}\text { Major depression- } \\
\text { CIDI-SF of the WHO }\end{array}$ & $\begin{array}{l}\text { MVA: Participants with } \\
\text { depression were more } \\
\text { likely to be non- } \\
\text { adherent than those } \\
\text { without a MI. } \\
(\mathrm{OR}=1.7,95 \% \\
\mathrm{CI}=1.3-2.3)\end{array}$ \\
\hline $\begin{array}{l}\text { Vranceanu, A. } \\
\text { M., S. } \\
\text { A. Safren, et al. } \\
(2008)\end{array}$ & $\begin{array}{l}\text { Randomized cohort } \\
\text { crossover trial } \\
\text { (Nov 2002-Jan } \\
\text { 2005; total of six } \\
\text { study visits, } \\
\text { enrollment and } \\
\text { five follow-up } \\
\text { visits) }\end{array}$ & $\begin{array}{l}156 \text { participants } \\
\text { Group 1: Two } \\
\text { physician visits } \\
\text { with } \\
\text { depression } \\
\text { screening then } \\
\text { crossover to } \\
\text { Group } 2 \\
\text { Group 2: Two } \\
\text { physician visits } \\
\text { without } \\
\text { depression } \\
\text { screening then } \\
\text { crossover to } \\
\text { Group } 1\end{array}$ & $\begin{array}{l}\text { M: EDMs adherence } \\
\text { D: Continuous variable } \\
\text { (percent adherence) } \\
\text { T: Previous } 30 \text { days } \\
\text { (which represents the } \\
\text { 30-day period after } \\
\text { depression } \\
\text { measurement) }\end{array}$ & $\begin{array}{l}\text { Depression- Brief (self- } \\
\text { report) screening } \\
\text { measures of depression } \\
\text { (PC-SAD) }\end{array}$ & $\begin{array}{l}\text { MVA: Continuous } \\
\text { Depression score was } \\
\text { significantly associated } \\
\text { with decreased percent } \\
\text { adherence }(p=0.015) \text {. } \\
\text { Dichotomous } \\
\text { depression score was } \\
\text { not }\end{array}$ \\
\hline $\begin{array}{l}\text { Waldrop- } \\
\text { Valverde, D. } \\
\text { and E. Valverde } \\
\text { (2005) USA }\end{array}$ & $\begin{array}{l}\text { Cross-sectional } \\
\text { study }\end{array}$ & $\begin{array}{l}58 \text { IDU } \\
\text { participants }\end{array}$ & $\begin{array}{l}\text { M: Self-report } \\
\text { D: } 100 \% \text { adherence } \\
\text { T: Previous day }\end{array}$ & Depression- BDI & $\begin{array}{l}\text { MVA: Depression was } \\
\text { significantly related to } \\
\text { decreasing adherence } \\
(\mathrm{OR}=0.924,95 \% \\
\mathrm{CI}=0.863-0.989 \\
p=0.023)\end{array}$ \\
\hline
\end{tabular}


Table 1 continued

\begin{tabular}{|c|c|c|c|c|c|}
\hline $\begin{array}{l}\text { Author, } \\
\text { publication year, } \\
\text { location }\end{array}$ & $\begin{array}{l}\text { Study design and } \\
\text { evaluation period }\end{array}$ & $\begin{array}{l}\text { PLWHA } \\
\text { population, } \\
\text { sample size }\end{array}$ & $\begin{array}{l}\text { Adherence: } \\
\text { measurement }(\mathrm{M}), \\
\text { definition }(\mathrm{D}) \text { and time } \\
\text { period }(\mathrm{T})\end{array}$ & $\begin{array}{l}\text { Depressive disorder and } \\
\text { scale used to measure } \\
\text { depressive disorder }\end{array}$ & $\begin{array}{l}\text { Impact of depressive } \\
\text { disorder on adherence }\end{array}$ \\
\hline $\begin{array}{l}\text { Wagner, G. J., K. } \\
\text { Goggin, et al. } \\
\text { (2011) USA }\end{array}$ & $\begin{array}{l}\text { Cross-sectional and } \\
\text { longitudinal } \\
\text { analyses of } 10 \\
\text { merged studies } \\
\text { (1997-2009) }\end{array}$ & $\begin{array}{l}1,374 \\
\text { participants }\end{array}$ & $\begin{array}{l}\text { M: EDMs (in all studies) } \\
\text { D: Continuous variable } \\
\text { and dichotomous } \\
\text { variable (good } \\
\text { adherence: }>90 \% \text { ) } \\
\text { T: Previous } 2 \text { weeks }\end{array}$ & $\begin{array}{l}\text { Depression-BDI }(\times 1) \text {, } \\
\text { BDI-II }(\times 4), \text { BSI }(\times 2) \\
\text { and CES-D }(\times 3)\end{array}$ & $\begin{array}{l}\text { MVA: In cross-sectional } \\
\text { multivariate analyses, } \\
\text { continuous depression, } \\
\text { cognitive depressive } \\
\text { symptoms, and severe } \\
\text { depression were } \\
\text { associated with lower } \\
\text { cART adherence. In } \\
\text { longitudinal analysis, } \\
\text { reductions in both } \\
\text { continuous and } \\
\text { categorical depression } \\
\text { predicted increased } \\
\text { cART adherence } \\
\text { (b }(\mathrm{SE})=-0.015, \\
p<0.01)\end{array}$ \\
\hline
\end{tabular}

II. Depressive disorder not significantly associated with adherence to cART

$\begin{array}{lc}\text { Berger- } & \text { Cross-sectional } \\ \text { Greenstein, J. } & \text { study } \\ \text { A., C. } & \\ \text { A. Cuevas, } & \\ \text { et al. (2007) } & \\ \text { USA } & \\ \text { Bottonari, K. A., } & \text { Prospective cohort } \\ \text { S. A. Safren, } & \text { study (3-month } \\ \text { et al. (2010) } & \text { follow-up) } \\ \text { USA } & \end{array}$

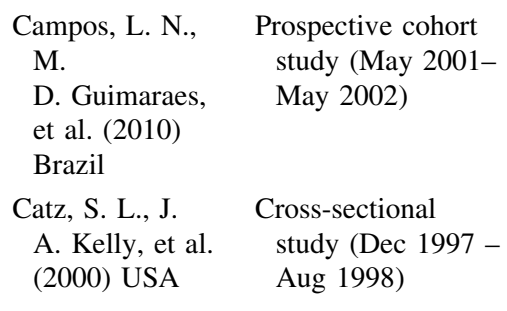

Gibbie, T., M. Longitudinal cohort 72 participants Hay, et al. (2007) Australia from Jan 2003 to Mar 2004;

$\begin{array}{ll}85 \text { participants } & \text { M: Self-report (ACTG- } \\ \text { diagnosed with } & \text { adapted questionnaire) } \\ \text { drug abuse \& } & \text { D: Continuous variable } \\ \text { psychiatric } & \text { T: Previous } 3 \text { days } \\ \text { disease } & \end{array}$

87 participants
M: Self-report (AACTG questionnaire)

D: Continuous variable; subsequent analyses utilized Box-Cox transformed variables to improve the normality of adherence

T: Previous 30 days (1 month)

293 participants $\quad$ M: Self-report

D: $\geq 95 \%$ adherence

T: Previous 3 days

72 participants
M: Self-report (7-point scale)

D: Non-adherence: $<100 \%$ adherence

T: Previous 5 days and 3 months

M: Self-report

D: $\geq 95 \%$ adherence

T: Previous 24 h, 4 days and 7 days followed-up for 24 months)
Depression-MINI and MADRAS

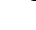
MDD-SCID \& BDI-II
DCID \& BDI-II

BVA: MDD was not significantly associated with non-adherence to cART
MVA: Neither depression nor depressive severity predicted HIV treatment adherence when controlling for the impact of acute life events

MVA: No significant
association was found
between non-
adherence and
depression
MVA: SCID diagnosis
of current major
depression was not
significantly related to
adherence ( $b=-0.99$,
$95 \%$
CI $=0.052-2.64$,
$p=0.322)$. BDI was
not included in the
analysis

Depression-HADS

Depression-CES-D

BVA: Depression was not independently associated with nonadherence

Depression- BDI (cutoff $=10$ ), and SCID 
Table 1 continued

\begin{tabular}{|c|c|c|c|c|c|}
\hline $\begin{array}{l}\text { Author, } \\
\text { publication year, } \\
\text { location }\end{array}$ & $\begin{array}{l}\text { Study design and } \\
\text { evaluation period }\end{array}$ & $\begin{array}{l}\text { PLWHA } \\
\text { population, } \\
\text { sample size }\end{array}$ & $\begin{array}{l}\text { Adherence: } \\
\text { measurement (M), } \\
\text { definition (D) and time } \\
\text { period (T) }\end{array}$ & $\begin{array}{l}\text { Depressive disorder and } \\
\text { scale used to measure } \\
\text { depressive disorder }\end{array}$ & $\begin{array}{l}\text { Impact of depressive } \\
\text { disorder on adherence }\end{array}$ \\
\hline $\begin{array}{l}\text { Gonzalez, J. S., F. } \\
\text { J. Penedo, et al. } \\
\text { (2004)USA }\end{array}$ & $\begin{array}{l}\text { Cross-sectional } \\
\text { study }\end{array}$ & $\begin{array}{l}90 \mathrm{HIV}(+) \\
\text { MSM and } \\
\text { women (of any } \\
\text { sexual } \\
\text { orientation) }\end{array}$ & $\begin{array}{l}\text { M: Self-report (ACTG } \\
\text { questionnaire) } \\
\text { D: Non-adherence: }<100 \\
\text { adherence } \\
\text { T: Previous } 4 \text { days }\end{array}$ & Depression-BDI & $\begin{array}{l}\text { MVA: BDI score was } \\
\text { not significantly related } \\
\text { to medication } \\
\text { adherence }\end{array}$ \\
\hline $\begin{array}{l}\text { Gordillo, V., J. } \\
\text { del Amo, et al. } \\
\text { (1999) Spain }\end{array}$ & $\begin{array}{l}\text { Cross-sectional } \\
\text { study (Dec } 1997 \text { - } \\
\text { May 1998) }\end{array}$ & 366 participants & $\begin{array}{l}\text { M: Self-report and pill } \\
\text { count method } \\
\text { (Returning those pills } \\
\text { that had not been taken } \\
\text { in the previous month } \\
\text { to the pharmacist) } \\
\text { D: }>90 \% \text { adherence } \\
\text { T: Previous } 1 \text { week and } \\
1 \text { month }\end{array}$ & $\begin{array}{l}\text { Depression-BDI, } \\
\quad(\text { cut-off }=14)\end{array}$ & $\begin{array}{l}\text { MVA: Depression was } \\
\text { not an independent } \\
\text { predictor of non- } \\
\text { adherence }\end{array}$ \\
\hline $\begin{array}{l}\text { Ingersoll, K. } \\
\text { (2004) USA }\end{array}$ & $\begin{array}{l}\text { Cross -sectional } \\
\text { study }\end{array}$ & 120 participants & $\begin{array}{l}\text { M1: Electronic medical } \\
\text { record and self-report } \\
\text { D: Four dichotomous } \\
\text { non-adherent } \\
\text { behaviors: [1] running } \\
\text { out of medications, [2] } \\
\text { not always taking } \\
\text { medications as } \\
\text { directed, [3] } \leq 95 \% \\
\text { adherence or [4] } \\
\text { having notations of } \\
\text { non-compliance in the } \\
\text { medical record } \\
\text { T: Previous week }\end{array}$ & $\begin{array}{l}\text { Major Depression -CIDI- } \\
\text { SF }\end{array}$ & $\begin{array}{c}\text { MVA: Major Depression } \\
\text { was not an independent } \\
\text { predictor of all four } \\
\text { non-adherent behaviors }\end{array}$ \\
\hline $\begin{array}{l}\text { Johnson, M. O., } \\
\text { S. E. Dilworth, } \\
\text { et al. (2011) } \\
\text { USA }\end{array}$ & $\begin{array}{l}\text { Cross-sectional } \\
\text { study (Jan 2009- } \\
\text { Sep 2010) }\end{array}$ & $\begin{array}{l}295 \text { HIV- } \\
\text { positive men } \\
\text { (data were } \\
\text { collected from } \\
210 \text { male } \\
\text { couples or } 420 \\
\text { men) }\end{array}$ & $\begin{array}{l}\text { M: Self-report (AACTG } \\
\text { questionnaire and the } \\
\text { visual analog (VAS) } \\
\text { scale) } \\
\text { D: } 100 \% \text { versus } \\
<100 \% \text { adherence } \\
\text { T: Previous } 3 \text { and } \\
30 \text { days }\end{array}$ & Depression-CES-D & $\begin{array}{l}\text { MVA: Depressive } \\
\text { symptoms were not } \\
\text { associated with } \\
\text { suboptimal 3-day or } \\
\text { 30-day adherence }\end{array}$ \\
\hline $\begin{array}{l}\text { Kalichman, S. C., } \\
\text { J. Pellowski, } \\
\text { et al.(2011) } \\
\text { USA }\end{array}$ & $\begin{array}{l}\text { Longitudinal cohort } \\
\text { study (Jan 2008- } \\
\text { Jun 2009) }\end{array}$ & 179 participants & $\begin{array}{l}\text { M: Unannounced pill } \\
\text { counts } \\
\text { D: Non-adherent: taking } \\
\quad<85 \% \text { of medications } \\
\text { T: For } 8 \text { consecutive } \\
\text { months }\end{array}$ & Depression-CES-D & $\begin{array}{l}\text { MVA: Depression was } \\
\text { not associated with } \\
\text { non- adherence }\end{array}$ \\
\hline $\begin{array}{l}\text { Keuroghlian, A. } \\
\text { S., C. } \\
\text { S. Kamen, et al. } \\
\text { 2011) USA }\end{array}$ & $\begin{array}{l}\text { Cross-sectional } \\
\text { study }\end{array}$ & 38 participants & $\begin{array}{l}\text { M: Self-report (AACTG } \\
\text { questionnaire) } \\
\text { D: Adherent: no missed } \\
\text { doses } \\
\text { T: Previous } 4 \text { days }\end{array}$ & Depression-CES-D & $\begin{array}{l}\text { MVA: Depression was } \\
\text { not significantly } \\
\text { associated with cART } \\
\text { adherence }(p>0.05)\end{array}$ \\
\hline $\begin{array}{l}\text { Kyser, M., K. } \\
\text { Buchacz, et al. } \\
\text { (2011) USA }\end{array}$ & $\begin{array}{l}\text { Cross-sectional } \\
\text { analysis of a } \\
\text { prospective cohort } \\
\text { study (The SUN } \\
\text { study, Mar } 2004 \text { - } \\
\text { Jun } 2006\end{array}$ & 528 participants & $\begin{array}{l}\text { M: Self-report } \\
\text { D: Non-adherence: } \\
\text { missing at least one } \\
\text { dose of medications } \\
\text { T: Previous } 3 \text { days }\end{array}$ & Depression-PHQ-9 & $\begin{array}{l}\text { BVA: Depression was } \\
\text { not an independent } \\
\text { predictor of non- } \\
\text { adherence }\end{array}$ \\
\hline
\end{tabular}


Table 1 continued

\begin{tabular}{|c|c|c|c|c|c|}
\hline $\begin{array}{l}\text { Author, } \\
\text { publication year, } \\
\text { location }\end{array}$ & $\begin{array}{l}\text { Study design and } \\
\text { evaluation period }\end{array}$ & $\begin{array}{l}\text { PLWHA } \\
\text { population, } \\
\text { sample size }\end{array}$ & $\begin{array}{l}\text { Adherence: } \\
\text { measurement (M), } \\
\text { definition (D) and time } \\
\text { period (T) }\end{array}$ & $\begin{array}{l}\text { Depressive disorder and } \\
\text { scale used to measure } \\
\text { depressive disorder }\end{array}$ & $\begin{array}{l}\text { Impact of depressive } \\
\text { disorder on adherence }\end{array}$ \\
\hline $\begin{array}{l}\text { Leserman, J., G. } \\
\text { Ironson, et al. } \\
\text { (2008) USA }\end{array}$ & $\begin{array}{l}\text { Cross-sectional } \\
\text { study (Feb 2004- } \\
\text { Feb 2007) }\end{array}$ & 105 participants & $\begin{array}{l}\text { M: Self-report (AACTG } \\
\text { questionnaire) } \\
\text { D: Non-adherence: } \\
\text { missing at least one } \\
\text { dose of medications } \\
\text { T: Previous } 2 \text { weeks }\end{array}$ & Depression—BDI & $\begin{array}{l}\text { MVA: Depressive } \\
\text { symptoms were not } \\
\text { associated with non- } \\
\text { adherence }\end{array}$ \\
\hline $\begin{array}{l}\text { Mellins, C. A., J. } \\
\text { F. Havens, et al. } \\
\text { (2009) USA }\end{array}$ & $\begin{array}{l}\text { Cross-sectional } \\
\text { study }\end{array}$ & 542 participants & $\begin{array}{l}\text { M: Self-report (AACTG } \\
\text { questionnaire) } \\
\text { D: } 100 \% \text { adherence } \\
\text { T: Previous } 3 \text { days }\end{array}$ & Major Depression -SCID & $\begin{array}{l}\text { BVA: The presence or } \\
\text { absence of major } \\
\text { depression on the } \\
\text { SCID was not } \\
\text { associated with } \\
\text { adherence }\end{array}$ \\
\hline $\begin{array}{l}\text { Mohammed, H., } \\
\text { L. Kieltyka, } \\
\text { et al. (2004) } \\
\text { USA }\end{array}$ & $\begin{array}{l}\text { Cross-sectional } \\
\text { study (Mar 1999- } \\
\text { Aug 2001) }\end{array}$ & 273 participants & $\begin{array}{l}\text { M: Self-report } \\
\text { D: Non-adherence: }<100 \\
\text { adherence } \\
\text { T: Previous week }\end{array}$ & $\begin{array}{l}\text { Depression-the } \\
\text { following questions: } \\
\text { "In the last } 7 \text { days did } \\
\text { you feel: [1] that you } \\
\text { could not shake the } \\
\text { blues? [2] depressed? } \\
\text { [3] fearful? and [4] that } \\
\text { your sleep had been } \\
\text { restless?" }\end{array}$ & $\begin{array}{l}\text { MVA: Depression was } \\
\text { not found to be } \\
\text { significantly associated } \\
\text { with non-adherence }\end{array}$ \\
\hline $\begin{array}{l}\text { Moore, D. J., C. } \\
\text { Posada, et al. } \\
\text { (2011) USA }\end{array}$ & $\begin{array}{l}\text { Cross-sectional } \\
\text { analysis of a } \\
\text { cohort study }\end{array}$ & 77 participants & $\begin{array}{l}\text { M: EDMs } \\
\text { D: Adherent: }>90 \% \\
\text { adherence } \\
\text { T: Previous } 30 \text { days }\end{array}$ & MDD-BDI & $\begin{array}{l}\text { BVA: Current or past } \\
\text { diagnosis of MDD was } \\
\text { not associated non- } \\
\text { adherence }(p>0.05)\end{array}$ \\
\hline $\begin{array}{l}\text { Moss, A. R., J. } \\
\text { A. Hahn, et al. } \\
\text { (2004) USA }\end{array}$ & $\begin{array}{l}\text { 12-month } \\
\text { prospective cohort } \\
\text { study (Mar } 1998 \\
\text { Apr 2001) }\end{array}$ & 148 participants & $\begin{array}{l}\text { M: Pill count self-report } \\
\text { and EDMs } \\
\text { D: [1] Continuous } \\
\text { variable } \\
\text { [2] cART } \\
\text { discontinuation: no } \\
\text { pills were taken for } \\
1 \text { month } \\
\text { T: Previous } 3 \text { days and } \\
1 \text { month }\end{array}$ & $\begin{array}{l}\text { Depressive symptoms- } \\
\text { BDI }(\text { score }>15)\end{array}$ & $\begin{array}{l}\text { BVA: BDI }>15 \text { was not } \\
\text { significantly associated } \\
\text { with cART } \\
\text { discontinuation or with } \\
\text { adherence }\end{array}$ \\
\hline $\begin{array}{l}\text { Mugavero, M., J. } \\
\text { Ostermann, } \\
\text { et al. (2006) } \\
\text { USA }\end{array}$ & $\begin{array}{l}\text { Cross-sectional } \\
\text { analysis (Dec } \\
\text { 2001-Apr 2002) }\end{array}$ & 474 participants & $\begin{array}{l}\text { M: Self-reported } \\
\text { D: Non-adherence: } \\
\quad<100 \% \text { adherence } \\
\text { T: Previous } 7 \text { days }\end{array}$ & Depression-BSI & $\begin{array}{l}\text { MVA: Depression was } \\
\text { not significantly } \\
\text { associated with non- } \\
\text { adherence (when } \\
\text { trauma variable was in } \\
\text { the model) }\end{array}$ \\
\hline $\begin{array}{l}\text { Palmer, N. B., J. } \\
\text { Salcedo, et al. } \\
\text { (2003) USA }\end{array}$ & $\begin{array}{l}\text { Cross-sectional } \\
\text { study }\end{array}$ & $\begin{array}{l}107 \text { participants } \\
\text { diagnosed with } \\
\text { substance } \\
\text { abuse and } \\
\text { psychiatric } \\
\text { diseases (all on } \\
\text { methadone) }\end{array}$ & $\begin{array}{l}\text { M: Self-report (AACTG } \\
\text { questionnaire) } \\
\text { D: } \geq 95 \% \text { adherence } \\
\text { T: Previous } 3 \text { days }\end{array}$ & Depression -SCID-I & $\begin{array}{l}\text { BVA: Depression was } \\
\text { not associated with } \\
\text { HIV medication } \\
\text { adherence }\end{array}$ \\
\hline $\begin{array}{l}\text { Shin, S., M. } \\
\text { Munoz, et al. } \\
\text { (2008) Peru }\end{array}$ & $\begin{array}{l}\text { Cross-sectional } \\
\text { study (Nov 2005- } \\
\text { Nov 2006) }\end{array}$ & $\begin{array}{l}43 \text { participants } \\
\text { with } \\
\text { tuberculosis }\end{array}$ & $\begin{array}{l}\text { M: Self-report } \\
\text { D: Non-adherence: } \\
\quad<100 \% \text { adherence } \\
\text { T: Previous } 4 \text { weeks }\end{array}$ & Depression-HSCL & $\begin{array}{l}\text { MVA: Depression by } \\
\text { HSCL (score >1.75) } \\
\text { was not significantly } \\
\text { associated with non- } \\
\text { adherence }\end{array}$ \\
\hline
\end{tabular}


Table 1 continued

\begin{tabular}{|c|c|c|c|c|c|}
\hline $\begin{array}{l}\text { Author, } \\
\text { publication year, } \\
\text { location }\end{array}$ & $\begin{array}{l}\text { Study design and } \\
\text { evaluation period }\end{array}$ & $\begin{array}{l}\text { PLWHA } \\
\text { population, } \\
\text { sample size }\end{array}$ & $\begin{array}{l}\text { Adherence: } \\
\text { measurement }(\mathrm{M}) \text {, } \\
\text { definition }(\mathrm{D}) \text { and time } \\
\text { period }(\mathrm{T})\end{array}$ & $\begin{array}{l}\text { Depressive disorder and } \\
\text { scale used to measure } \\
\text { depressive disorder }\end{array}$ & $\begin{array}{l}\text { Impact of depressive } \\
\text { disorder on adherence }\end{array}$ \\
\hline $\begin{array}{l}\text { Van Servellen, } \\
\text { G., B. Chang, } \\
\text { et al. (2002) } \\
\text { USA }\end{array}$ & $\begin{array}{l}\text { Cross-sectional } \\
\text { study }\end{array}$ & 182 participants & $\begin{array}{l}\text { M: Self-report and } \\
\text { medical records } \\
\text { D: Non-adherence: self- } \\
\text { report or presence of } \\
\text { non-adherence } \\
\text { behavior in the medical } \\
\text { records } \\
\text { T: Previous } 3 \text { months }\end{array}$ & Depression-HADS & $\begin{array}{l}\text { MVA: Depression was } \\
\text { not shown to be } \\
\text { associated with non- } \\
\text { adherence }\end{array}$ \\
\hline $\begin{array}{l}\text { Wagner, G. J., L. } \\
\text { M. Bogart, et al. } \\
\text { (2011) USA }\end{array}$ & Cohort study & $\begin{array}{l}214 \text { African } \\
\text { American males }\end{array}$ & $\begin{array}{l}\text { M: EDMs } \\
\text { D: Continuous variable } \\
\text { T: Previous } 6 \text { months }\end{array}$ & $\begin{array}{l}\text { Depression: } 8 \text {-item } \\
\text { depression scale from } \\
\text { the Medical Outcomes } \\
\text { Study }\end{array}$ & $\begin{array}{l}\text { BVA: Depression was } \\
\text { not associated with } \\
\text { adherence }\end{array}$ \\
\hline \multicolumn{6}{|c|}{ III. Adherence variable predicts change in depressive symptoms } \\
\hline $\begin{array}{l}\text { Ammassari, A., } \\
\text { A. Antinori, } \\
\text { et al. (2004) } \\
\text { Italy }\end{array}$ & $\begin{array}{l}\text { Multicenter Cross- } \\
\text { sectional study } \\
\text { (Nov 1999-Feb } \\
\text { 2000) }\end{array}$ & 135 participants $^{\mathrm{a}}$ & $\begin{array}{l}\text { M: Self-report } \\
\text { D: Non-adherence: } \\
\text { Missing at least } 1 \text { dose } \\
\text { of cART } \\
\text { T: Previous } 1 \text { week }\end{array}$ & $\begin{array}{l}\text { Depression-MADRAS } \\
\quad(\text { score }>19)\end{array}$ & $\begin{array}{l}\text { MVA: Depression scores } \\
\text { were significantly } \\
\text { higher in the non- } \\
\text { adherent group, } \\
\text { compared with the } \\
\text { adherent group } \\
(p=0.03)\end{array}$ \\
\hline $\begin{array}{l}\text { Bianco, J. A., T. } \\
\text { G. Heckman, } \\
\text { et al. (2011) } \\
\text { USA }\end{array}$ & $\begin{array}{l}\text { Cross-sectional } \\
\text { study } \\
\text { (Jun 2008-Jul 2009) }\end{array}$ & $\begin{array}{l}242 \text { participants } \\
(>50 \text { years old } \\
\text { adults enrolled } \\
\text { in a RCT who } \\
\text { had a score of } \\
>5 \text { on the } \\
\text { GDS })\end{array}$ & $\begin{array}{l}\text { M: Self-report (ACTG } \\
\text { questionnaire) } \\
\text { D: Non-adherent: } \\
\text { skipping medication at } \\
\text { least once and/or } \\
\text { taking a medication } \\
\text { late at least twice } \\
\text { T: Previous } 7 \text { days }\end{array}$ & $\begin{array}{l}\text { Depression-Geriatric } \\
\text { Depression Scale } \\
(\text { GDS })\end{array}$ & $\begin{array}{l}\text { MVA: Non-adherent } \\
\text { participants were } \\
\text { significantly more } \\
\text { depressed }(b=-0.05 \text {, } \\
\text { Wald's } \chi^{2}=4.52, \\
p<0.033)\end{array}$ \\
\hline $\begin{array}{l}\text { Catz, S. L., T. } \\
\text { G. Heckman, } \\
\text { et al. (2001) } \\
\text { USA }\end{array}$ & $\begin{array}{l}\text { Cross-sectional } \\
\text { study (1997) }\end{array}$ & 84 participants & $\begin{array}{l}\text { M: Self-report (six-point } \\
\text { Likert scale) } \\
\text { D: Dichotomized as } \\
\text { 'consistent adherence' } \\
\text { (No skipped doses) or } \\
\text { 'inconsistent } \\
\text { adherence' (at least } 1 \\
\text { skipped dose) } \\
\text { T: Previous } 7 \text { days }\end{array}$ & Depression -BDI & $\begin{array}{l}\text { BVA: Adherence was } \\
\text { not associated with } \\
\text { depression }\end{array}$ \\
\hline $\begin{array}{l}\text { Farley, J., E. } \\
\text { Miller, et al. } \\
\text { (2010) Nigeria }\end{array}$ & $\begin{array}{l}\text { Cross-sectional } \\
\text { study (June-July } \\
\text { 2007) }\end{array}$ & $\begin{array}{l}399 \text { participants } \\
\text { (222 cART- } \\
\text { experienced } \\
\text { and } 177 \text { cART- } \\
\text { naive) }\end{array}$ & $\begin{array}{l}\text { M: Pharmacy refill } \\
\text { adherence rate } \\
\text { D: Non-adherence: } \\
\text { pharmacy refill rate } \\
<95 \% \\
\text { T: From the time cART } \\
\text { was first dispensed } \\
\text { until a cutoff date } \\
\text { shortly after the study } \\
\text { ended }\end{array}$ & $\begin{array}{l}\text { Depression -CES-D } \\
\text { Binary cut-off for CES- } \\
\text { D scores are defined as } \\
\geq 16 \text { and } \geq 21\end{array}$ & $\begin{array}{l}\text { MVA: Having a } \\
\text { pharmacy refill rate } \\
<95 \%, \text { was associated } \\
\text { with a CES-D } \geq 16 \\
(p=0.004) \text { and a } \\
\text { CES-D } \geq 21 \\
(p<0.001)\end{array}$ \\
\hline $\begin{array}{l}\text { Herrmann, S., E. } \\
\text { McKinnon, } \\
\text { et al. (2008) } \\
\text { Australia }\end{array}$ & $\begin{array}{l}\text { Longitudinal cohort } \\
\text { study (Jul } \\
\text { 2003-Dec 2005; } \\
>6 \text { months } \\
\text { follow-up) }\end{array}$ & 357 participants & $\begin{array}{l}\text { M: Self-report (AACTG } \\
\text { questionnaire) } \\
\text { D: } 100 \% \text { adherence } \\
\text { T: Previous month }\end{array}$ & $\begin{array}{l}\text { Depression-CES-D scale } \\
\text { (depression used as } \\
\text { dependent variable) }\end{array}$ & $\begin{array}{l}\text { BVA: Non-adherent } \\
\text { participants scored } \\
\text { higher values on the } \\
\text { depression indicator } \\
\text { scale }(p=0.03)\end{array}$ \\
\hline
\end{tabular}


Table 1 continued

\begin{tabular}{|c|c|c|c|c|c|}
\hline $\begin{array}{l}\text { Author, } \\
\text { publication year, } \\
\text { location }\end{array}$ & $\begin{array}{l}\text { Study design and } \\
\text { evaluation period }\end{array}$ & $\begin{array}{l}\text { PLWHA } \\
\text { population, } \\
\text { sample size }\end{array}$ & $\begin{array}{l}\text { Adherence: } \\
\text { measurement (M), } \\
\text { definition (D) and time } \\
\text { period (T) }\end{array}$ & $\begin{array}{l}\text { Depressive disorder and } \\
\text { scale used to measure } \\
\text { depressive disorder }\end{array}$ & $\begin{array}{l}\text { Impact of depressive } \\
\text { disorder on adherence }\end{array}$ \\
\hline $\begin{array}{l}\text { Springer, S. A., S. } \\
\text { Chen, et al. } \\
\text { (2009) USA }\end{array}$ & 6-month RCT & $\begin{array}{l}89 \text { IDU } \\
\text { participants }\end{array}$ & $\begin{array}{l}\text { M: Self-report (ACTG } \\
\text { questionnaire) } \\
\text { D: Continuous variable } \\
\text { (reported as a mean } \\
\text { change in percentage } \\
\text { adherence) } \\
\text { T: Previous } 3 \text { days }\end{array}$ & $\begin{array}{l}\text { Depression-CES-D, } \\
\text { MDD was defined as } \\
\text { having a CES-D score } \\
>16 \\
\text { (depression used as } \\
\text { dependent variable) }\end{array}$ & $\begin{array}{l}\text { MVA: Adherence to } \\
\text { cART was associated } \\
\text { with improved } \\
\text { depressive symptoms. } \\
\text { Increased adherence } \\
\text { was significantly } \\
\text { associated to decreased } \\
\text { CES-D score } \\
(p=0.01)\end{array}$ \\
\hline
\end{tabular}

$I V$. Depressive disorder associated with decreased persistence on cART

\begin{tabular}{|c|c|c|c|c|c|}
\hline $\begin{array}{l}\text { Carrico, A. W., E. } \\
\text { D. Riley, et al. } \\
\text { (2011) USA }\end{array}$ & $\begin{array}{r}\text { Cohort study (using } \\
\text { data from a RCT) }\end{array}$ & 603 participants & $\begin{array}{l}\text { M: Self-report } \\
\text { D: cART utilization } \\
\text { classified as: [1] } \\
\text { continuous cART } \\
\text { utilization-being on } \\
\text { cART at baseline and } \\
\text { remaining on cART; } \\
\text { [2] cART } \\
\text { discontinuation- } \\
\text { stopping cART and } \\
\text { remaining off during } \\
\text { any subsequent follow- } \\
\text { up assessments; and [3] } \\
\text { intermittent cART } \\
\text { utilization-stopping } \\
\text { and restarting cART at } \\
\text { least once } \\
\text { T: } 25 \text { months of follow- } \\
\text { up }\end{array}$ & Depression-BDI-I & $\begin{array}{l}\text { MVA: Depression at } \\
\text { baseline independently } \\
\text { predicted a } 39 \% \\
\text { increase in the odds of } \\
\text { cART discontinuation } \\
(\mathrm{OR}=1.39,95 \% \\
\mathrm{CI}=1.08-1.78, \\
p<0.01)\end{array}$ \\
\hline $\begin{array}{l}\text { Li, X., J. } \\
\text { B. Margolick, } \\
\text { et al. (2005) } \\
\text { USA }\end{array}$ & $\begin{array}{r}\text { Cohort study (Apr } \\
\text { 1999-Mar 2002) }\end{array}$ & 687 MSM & $\begin{array}{l}\text { M: Self-report (of cART } \\
\text { persistence) } \\
\text { D: [1] Continuing } \\
\text { cART - not stopping } \\
\text { medications for } 2 \text { or } \\
\text { more consecutive days } \\
\text { [2] Interrupted } \\
\text { cART-stopping } \\
\text { cART for }>2 \\
\text { consecutive days at } \\
\text { least once; and [3] } \\
\text { Discontinued cART- } \\
\text { stopping all ART } \\
\text { between and still off } \\
\text { cART at end of } \\
\text { assessment period } \\
\text { T: Previous } 6 \text { months } \\
\text { (time between Vi and } \\
\text { Vi+1) }\end{array}$ & Depression—CES-D & $\begin{array}{l}\text { MVA: CES-D score }>16 \\
\text { was an independent } \\
\text { predictor for } \\
\text { Interrupting cART, } \\
(\mathrm{OR}=1.97,95 \% \\
\mathrm{CI}=1.38-2.80) \text {, and } \\
\text { Discontinuing cART, } \\
(\mathrm{OR}=2.03,95 \% \\
\mathrm{CI}=1.24-3.32) \text { but } \\
\text { not for continuing } \\
\text { cART }\end{array}$ \\
\hline
\end{tabular}


Table 1 continued

\begin{tabular}{|c|c|c|c|c|c|}
\hline $\begin{array}{l}\text { Author, } \\
\text { publication year, } \\
\text { location }\end{array}$ & $\begin{array}{l}\text { Study design and } \\
\text { evaluation period }\end{array}$ & $\begin{array}{l}\text { PLWHA } \\
\text { population, } \\
\text { sample size }\end{array}$ & $\begin{array}{l}\text { Adherence: } \\
\text { measurement (M), } \\
\text { definition (D) and time } \\
\text { period }(\mathrm{T})\end{array}$ & $\begin{array}{l}\text { Depressive disorder and } \\
\text { scale used to measure } \\
\text { depressive disorder }\end{array}$ & $\begin{array}{l}\text { Impact of depressive } \\
\text { disorder on adherence }\end{array}$ \\
\hline $\begin{array}{l}\text { Maru, D. S., R. } \\
\text { D. Bruce, et al. } \\
\text { (2008) USA }\end{array}$ & $\begin{array}{l}\text { 6-month RCT of } 2: 1 \\
\text { to DAART versus } \\
\text { SAT }\end{array}$ & 141 drug users & $\begin{array}{l}\text { M: EDMs, observed } \\
\text { doses, and self-report. } \\
\text { Both persistence and } \\
\text { adherence were } \\
\text { measured } \\
\text { D: The time-to-drop-out } \\
\text { (persistence) was } \\
\text { considered to be the } \\
\text { duration from the first } \\
\text { observed dose of } \\
\text { DAART to the last } \\
\text { DAART visit the } \\
\text { participant received } \\
\text { T: Previous } 6 \text { months }\end{array}$ & Depression-CES-D & $\begin{array}{l}\text { BVA: The presence of } \\
\text { severe score on the } \\
\text { CES-D scale predicted } \\
\text { time-to-DAART } \\
\text { discontinuation } \\
(\mathrm{HR}=2.4,95 \% \\
\mathrm{CI}=1.0-6.0, \text { Gehan } \\
\text { statistic }=4.4 \text {; } \\
p<0.05)\end{array}$ \\
\hline \multicolumn{6}{|c|}{ V. Depressive disorder associated with increased persistence on cART } \\
\hline $\begin{array}{l}\text { Himelhoch, S., C. } \\
\text { H. Brown, et al. } \\
\text { (2009) USA }\end{array}$ & $\begin{array}{l}\text { Longitudinal cohort } \\
\text { study (2000-2005) }\end{array}$ & $\begin{array}{l}4989 \\
\text { participants }\end{array}$ & $\begin{array}{l}\text { M: Self-report } \\
\text { D: cART } \\
\text { discontinuation: the } \\
\text { participant either went } \\
\text { off cART but remained } \\
\text { active in care or } \\
\text { dropped out of active } \\
\text { care } \\
\text { T: Previous year }\end{array}$ & $\begin{array}{l}\text { Depressive disorder was } \\
\text { defined using ICD-9 }\end{array}$ & $\begin{array}{l}\text { MVA: The hazard } \\
\text { probability for cART } \\
\text { discontinuation among } \\
\text { those with depressive } \\
\text { disorders was } \\
\text { significantly lower in } \\
\text { the first year } \\
\text { (AOR }=0.61,95 \% \\
\text { CI }=0.54-0.69 \text { ); but it } \\
\text { did not significantly } \\
\text { differ in subsequent } \\
\text { years }\end{array}$ \\
\hline
\end{tabular}

AACTG Adult AIDS Clinical Trials Group, ADT Anti-Depressant Therapy, AOR Adjusted Odds Ratio, BDI (-II) Beck Depression Index (2nd Edition), BSI Brief Symptom Inventory, BVA Bivariate Analysis, CES-D Center for Epidemiologic Studies-Depression Scale, CIDI-SF Composite International Diagnostic Interview-Short Form, DAART Directly Administered Antiretroviral Therapy, GDS Geriatric Depression Scale, $c A R T$ combination Antiretroviral Therapy, HADS Hospital Anxiety and Depression Scale, HSCL Hopkins Symptom Checklist-15, HR Hazard Ratio, ICD-9-CM International Classification of Diseases, 9th Revision, Clinical Modification, IDU Injection Drug User, IES Impact of Event Scale, IRR Incidence Rate Ratio, MADRAS Montgomery-Asberg Depression Rating Scale, EDMs Electronic Drug Monitors, MDD Major Depressive Disorder, MSM Men who have sex with men, MINI Mini International Neuropsychiatric Interview, MVA Multivariate analysis, OR Odds Ratio, PLWHA People Living with HIV/AIDS, POMS Profile of Mood States depression factor scale, PHQ-9 9- item Patient Health Questionnaire, $R C T$ Randomized Control Trials, $R H$ Risk Hazard ratio, RR Relative Risk, SAT Self-administered therapy, SCAN Schedule for Clinical Assessment in Neuropsychiatry, SCID-IV Structured Clinical Interview for DSM-IV, SD Standard Deviation, VAS Visual Analog Scale

${ }^{a}$ Participants: People Living with HIV/AIDS (PLWHA) $>18$ years old

from: 1 day to 4 weeks; 7 days (the most commonly used recall period) ( $N=22$ studies $) ; 4$ weeks ( $N=12$ studies $)$; 4 days ( $N=12$ studies); 3 days ( $N=10$ studies); 2 days ( $N=6$ studies); 3 months ( $N=4$ studies); 1 day $(N=4$ studies); 2 weeks ( $N=3$ studies), and 5 days $(N=1$ study). Adherence was reported either as a continuous or binary (dichotomous) measure, either in association with mental disorders or as the proportion of the sample meeting a specified level of adherence. There was considerable variation for binary (dichotomous) adherence measure and its cut-off, differentially defined by studies as $\geq 75 \%$ adherence, $\geq 80 \%$ adherence, $\geq 90 \%$ adherence, $\geq 95 \%$ adherence, $100 \%$ adherence, no missed doses, or a specific number of missed doses for a given period of time.

\section{Antiretroviral Persistence Measurements}

Among five studies that measured cART regimen persistence, four used self-report measures and one used EDMs, self-report, and DOT combined. The time period over which persistence was assessed ranged from 6 months to 3 years.

Measures of Mental Disorders

In addition to ICD-9, ICD-10 and DSM-IV diagnostic criteria, other validated and non-validated screening tools (see Table 1 in the manuscript and Tables 2 and 3 in the electronic appendix) were used to screen for and diagnose 
specific mental disorders and evaluate mental disorder symptoms. The most commonly evaluated mental disorder was depressive disorder $(N=62)$, followed by anxiety disorders $(N=17)$, bipolar disorder $(N=5)$, psychotic disorders $(N=3)$, personality disorders $(N=2)$, and adjustment disorder $(N=2)$. Some studies evaluated more than one mental disorder and used more than one instrument to evaluate the presence of one or more mental disorders. Depressive disorder and depressive symptoms were measured by using one or two self-report scales in 60 studies whereas two studies used a diagnosis of depression (ICD-9 code) on participants' medical charts. The most common instrument employed to assess depression and depressive symptoms was the Center for Epidemiological Studies Depression Scale (CES-D) [45, 46], $(N=23$; $37 \%$ ) followed by the Beck Depression Inventory (BDI and BDI-II) [47-50] $(N=18 ; 29 \%)$ and the Structured Clinical Interview for DSM-IV (SCID) [51] (four studies; $6 \%$ ), with the remaining studies using seventeen other scales: Montgomery-Asberg Depression Rating Scale $(N=3)$ [52], Brief Symptom Inventory (BSI) $(N=3)$ [53], Hospital Anxiety and Depression Scale (HADS) $(N=2)$, Composite International Diagnostic InterviewShort Form (CIDI-SF), Mini International Neuropsychiatric Interview (MINI) $(N=2)$, ICD-9 $(N=2)$, Hopkins Symptom Checklist-15, Burnam's interviewer administered 8-item screening tool, Schedule for Clinical Assessment in Neuropsychiatry (SCAN), Profile of Mood States (POMS) depression factor scale, Brief (self-report) screening measures of depression (PC-SAD) [54], Geriatric Depression Scale (GDS) [55], Patient Health Questionnaire (PHQ-9) $(N=2)$ [56], the 8-item depression scale from the Medical Outcomes Study, the 15-item screening test developed and used previously in Thailand (Thai Department of Mental Health, 2006), and unspecified instruments constructed from 3 to 4 questions ( $N=2$ studies) [57, 58].

\section{Primary Outcomes Analysis}

Two outcomes were evaluated in this systematic review: (a) cART adherence and (b) cART persistence. Adherence and persistence data for each article were extracted and are presented in Tables 1 in the manuscript; and Tables 2, 3, and 4 in the electronic appendix. The following sections are grouped based on each mental disorder and the effect on these two outcomes.

\section{Adherence to cART}

Impact of Depressive Disorders on cART Adherence Fifty-eight studies evaluated the impact of depressive disorder (as either the dependent or independent variable) on cART adherence. These data are presented in Table 1.

Depressive Disorders Associated with Decreased Adherence to $c A R T$. Depressive disorders were significantly associated with decreased cART adherence in thirty out of 52 studies (57\%). Among these thirty studies, thirteen were cohort [59-71], one was a randomized control trial (RCT) [72], and 16 were cross-sectional studies [29, 57, 73-86]. The majority of these studies used selfreport measures for assessing the cART adherence variable $(N=25 / 30,83 \%)$, while the other five studies used EDMS, pharmacy refill data and unannounced pill counts to assess adherence. Depression symptom measures were used in the majority of these studies to assess the variable 'depression', with the CES-D $(N=13)$ and the BDI $(N=7)$ as the most common measures. Twenty-seven of the thirty studies $(90 \%)$ used multivariate analysis to evaluate the association with depressive symptoms/diagnosis and poor cART adherence.

Depressive Disorders not Significantly Associated with Adherence to cART. Twenty-two studies did not find a statistically significant association between depression and decreased cART adherence. Sixteen were cross-sectional [34-36, 58, 87-98], whereas six were cohort studies [38, 99-103]. The majority of these studies utilized a diagnosis of depressive disorder at baseline (either via the SCID, MINI, CIDI, HADS, or ICD-9 code) as the depression variable $(N=10)$, while seven studies used a depressive symptom scale, and the remainder of the studies utilized other non-standardized measures to determine depressive symptoms/disorder, such as the HSCL and a non-specified scale. Multivariate analyses were reported for finding an association with depressive disorder or depressive symptoms and cART adherence in the majority of these studies. The majority of studies utilized self-report measures for the adherence variable.

Adherence Variable Predicts Depressive Symptoms. In six studies, depression was the dependent variable while adherence to cART was the independent variable [104109]. Five of these studies reported an association with non-adherence to cART and higher depressive symptoms [104-108]. The one study that did not find a statistically significant association between cART adherence and depression had a relatively smaller sample size $(N=84)$ as compared to the studies that did find an association, was completed earlier than the other studies (year 1997), and only used a six-point likert scale to measure adherence [109].

Impact of Anxiety Disorders on cART Adherence Table 2 lists seventeen articles that evaluated the impact of one or more anxiety disorders on cART adherence. Anxiety disorders included: (i) unspecified anxiety disorder $(N=8)$, 
(ii) generalized anxiety disorder $(\mathrm{GAD}, N=3)$, (iii) posttraumatic stress disorder (PTSD, $N=9$ ), (iv) panic disorder $(N=3)$, (v) agoraphobia $(N=1)$, or a combination of the above (see Table 2 in the electronic appendix).

Impact of PTSD on cART Adherence. The relationship between PTSD and cART adherence was examined by nine studies (four cohort, and five cross-sectional studies). Two studies (one prospective cohort and one cross-sectional) found that PTSD was significantly associated with decreased cART adherence [33, 34]. Both of these studies utilized self-report adherence measures and examined results with multivariate analyses. One of these studies used the Post-Traumatic Diagnostic Scale (PDS) and the Impact of Event Scale (IES) while the other study used the IES only.

In one study, PTSD participants were significantly more likely to adhere to cART than depressed participants. In this study, 69 participants diagnosed with PTSD or depression were classified into four groups according to the severity of symptoms: (i) control (low PTSD/low depression); $N=22$; (ii) PTSD (high PTSD/low depression), $N=11$; (iii) depressed (low PTSD/high depression), $N=12$ ); and (iv) mixed (high $\mathrm{PTSD} /$ high depression), $N=24)$. The PTSD group was significantly more likely to adhere to cART regimens compared to the depressed group during the previous week $(\mathrm{OR}=23.9,95 \% \mathrm{CI}=$ 1.607-356.075) and during the previous 2 weeks of the study $(\mathrm{OR}=27.55 ; 95 \% \mathrm{CI}=1.99-381.82)$ [110].

Six studies (three cross-sectional, two cohort and one RCT) found no significant association between PTSD and cART adherence [36, 59, 72, 94, 103, 111]. Adherence was measured using self-report instruments in five studies and EDM in one study. PTSD was measured using the SCID $(N=2)$, PDS $(N=2)$, IES $(N=1)$ and a short form of the widely used Davidson Trauma Scale (SPAN, $N=1$ ).

Impact of Panic Disorder on cART Adherence. Three studies examined the effect of panic disorder on cART adherence. A study of 1,910 participants found that participants with panic disorder were more likely to be nonadherent to cART than those who were not diagnosed with a mental disorder after being screened with the full CIDI [68]. In the remaining two cross-sectional studies, panic disorder diagnosed using the SCID-I was not significantly associated with cART adherence [36, 94]. These two studies used self-report instruments to measure adherence to cART.

Impact of Generalized Anxiety Disorder (GAD) on cART Adherence. The impact of GAD on cART adherence was assessed in three reviewed articles.

One study reported that participants with GAD were more likely to be non-adherent than those without a mental disorder [68]. This cohort study of persons with a diagnosis of GAD measured adherence by the ACTG 7-day recall method, while the other two articles that found no significant association between GAD and cART adherence were cross-sectional evaluations and used the ACTG 3-day recall method [36, 94]. In one of these studies the participants had to have associated panic disorder in addition to the GAD, therefore making the specific association with GAD difficult to assess.

Impact of Other Anxiety Disorders (Agoraphobia, Unspecified Anxiety Disorder) on cART Adherence. Agoraphobia was evaluated as a separate disorder in one crosssectional study of 542 participants, and was not found to be significantly associated with cART adherence [36]. This study used a self-report instrument to measure adherence to cART and agoraphobia was diagnosed using the SCID.

Eight studies examined the association between an unspecified anxiety disorder (defined as either anxiety symptoms or anxiety disorders in general) and cART adherence. In three articles, anxiety symptoms were significantly associated with decreased cART adherence [10, $38,111]$. In these three studies, adherence was measured using self-report instruments $(N=2)$ and pharmacy refill $(N=1)$. Anxiety symptoms were measured using the Hospital Anxiety and Depression Scale (HADS, $N=1$ ), State-Trait Anxiety Inventory (STAI, $N=1$ ), and BSI $(N=1)$. One of these studies reported the result based on multivariate analysis and two of them reported the results based on bivariate analysis. Four studies found no statistically significant association between anxiety and non-adherence [60, 83, 96, 109]. In these four studies, adherence was measured using self-report instruments. Anxiety symptoms were measured using Hospital Anxiety and Depression Scale (HADS, $N=1$ ), State-Trait Anxiety Inventory (STAI, $N=1$ ), Symptom Check List-90Revised $(N=1)$, and a self-administered questionnaire/ face-to-face interview of somatic symptoms of anxiety $(N=1)$. Three of these studies reported the result based on multivariate analysis whereas only one of them reported the results based on bivariate analysis. In another study of unspecified anxiety disorder, anxiety symptoms significantly predicted that participants adhered to cART [91]. In this cross-sectional study of 120 participants with nonspecific anxiety symptoms measured with the CIDI-SF scale, cART adherence was measured using four dichotomous non-adherent questions based on electronic medical records.

Impact of Psychotic Disorder (Schizophrenia) on cART Adherence Two cross-sectional studies did not find a statistically significant association between schizophrenia/ psychotic disorder and cART adherence [94, 112]. In one of these studies the presence of a psychotic disorder among mentally ill participants was confirmed by a referring 
mental health professional, while in the other study the presence of psychotic disorder among triply diagnosed participants with HIV, substance abuse and mental disorders was confirmed using the SCID. Both studies used selfreport measures to evaluate adherence to cART, however one study assessed this with the ACTG 3-Day recall and the other used self report and EDMs.

Impact of Personality Disorders on cART Adherence Two cross-sectional studies examined the association between personality disorders (borderline and antisocial) and cART adherence. Both of these studies utilized the self-report ACTG 3-day recall adherence measure, but they differed in the subject populations. One study of HIV+ persons enrolled in multi-site cohort study found no statistically significant association between borderline or antisocial personality disorders and adherence [36]. The other study of $107 \mathrm{HIV}+$ participants with cooccurring substance use disorders enrolled in a methadone program found that borderline personality disorder was associated with non-adherence to cART, while antisocial personality disorder was not [94].

Impact of Bipolar Disorder on cART Adherence The effect of having bipolar disorder on cART adherence was examined in four cross-sectional studies. One study reported that bipolar disorder was significantly associated with decreased adherence to cART [35] and utilized EDMs as the adherence tool. In three studies, bipolar disorder was not significantly associated with decreased adherence to cART [36, 94, 112]. Adherence tools and subject populations differed between these three studies.

Impact of Somatization, Dissociation, Adjustment Disorder and Unspecified Mental Disorders on cART Adherence One study found that levels of somatization uniquely predicted adherence problems in a multivariate model [109]. Another study found that dissociation moderated the effect of PTSD on adherence resulting in lower odds of cART adherence (OR $=00.95, p<0.05)$. PTSD symptoms were significantly associated with lower odds of adherence in individuals reporting high levels of dissociation (OR $=0.86, p<0.05)$ but not in those reporting low levels of dissociation ( $\mathrm{OR}=1.02, p>0.05)$ [34].

Two cross-sectional studies found no significant association between adjustment disorder and cART adherence $[36,94]$. Both of these studies utilized the ACTG 3 -day recall for cART adherence assessment.

Six studies included in this systematic review assessed the impact of unspecified mental disorders on adherence to cART. In these papers, mental disorder was either used as a general term or combined with more than one mental disorder into one variable (see Table 3 in the electronic appendix). All six studies (four cross-sectional and two cohort) reported a significant association between the mental disorder and decreased adherence to cART [6, 113117]. Adherence was measured using self-report instruments in five studies and EDMs in one study. Five different instruments were used to measure the presence of a mental disorder (see Table 3 in the electronic appendix).

\section{Persistence on cART}

Impact of Depressive Disorder on cART Persistence Three studies, two longitudinal cohort and one RCT, found that depression was associated with decreased cART persistence $[31,118,119]$. Another study found less cART discontinuation in the first year among those with depressive disorder, but there was no association with cART persistence after the first year [120].

Impact of Bipolar Disorder on cART Persistence One cohort study concluded that HIV+ Medicaid beneficiaries with severe affective disorder (defined as having bipolar disorder plus major depressive disorder identified by ICD-9 codes) were significantly less persistent in their use of cART than those without serious mental illness [121].

Impact of Psychotic Disorder on cART Persistence In one cohort study, psychotic disorder (schizophrenia) identified by ICD-9 coding was not significantly associated with persistence on cART [121].

Impact of Unspecified Mental Disorder on cART Persistence One cohort study of 4,989 PLWHA found that compared to participants with no mental disorders, the hazard probability for discontinuing cART was significantly lower in the first and second years of treatment among those with a severe mental disorder (defined as ICD-9 codes for schizophrenia, other psychoses and bipolar disorder). Among participants with mental disorders, those with six or more mental health visits per year were significantly less likely to discontinue cART compared with participants with no mental health visits [120].

Impact of Antidepressant Treatment on Adherence to cART

Nine studies (seven cohort studies, one RCT, and one cross-sectional study) evaluated the impact of pharmacologic ADT on adherence to cART among depressed PLWHA (see Table 4 in the electronic appendix). Seven studies reported that pharmacologic treatment for depression was significantly associated with increased cART adherence [69, 122-127], while in one study ADT of depression was associated with decreased cART adherence. In this study, however, pharmacotherapy was NOT 
specifically evaluated. The authors defined treatment of depression as: 'seeing a psychiatrist, receiving a diagnosis of depression, or being prescribed ADT' [11]. Therefore, the construct validity of this study is problematic since the employed definition of psychiatric treatment did not distinguish the treatment of depression from a diagnosis of depression.

A RCT reported that participants receiving directly observed fluoxetine for the treatment of depressive symptoms had similar cART adherence compared to participants in the referral arm [128]. This study, however, had high degrees of continuation of cART at the end of the study in both arms (73 vs $75 \%$ ), suggesting an overall high degree of persistence on therapy.

\section{Discussion}

DSM-IV mental disorders, especially depressive disorders, are extremely common among PLWHA and have been associated with decreased adherence to cART and increased mortality [29, 32, 57, 73]. The literature concerning HIV and mental disorders is staggering, yet the data has yet to be rigorously appraised. Although a metaanalysis studying the effect of depression on cART adherence was recently published [37], this is to our knowledge the first systematic review to examine the impact of all mental disorders, with the exception of substance use disorders, on cART adherence and persistence among PLWHA.

The majority of papers included in this review studied the impact of depression on cART adherence and persistence. Thirty of the 52 studies $(57 \%)$ that studied adherence as the dependent variable, five out of six studies $(83 \%)$ that studied adherence as the independent variable, and three out of the four studies $(75 \%)$ that examined persistence reported that a depressive disorder was associated with a decrease in cART adherence and persistence. Among these studies, $90 \%$ based their results on multivariate analysis compared to $60 \%$ of the studies that did not find an association between depression and cART adherence and persistence. One study reported a decreased probability of cART discontinuation among those with depressive disorders in the first year but not in subsequent years [120]. This study, however, did not measure adherence in itself, but rather cART discontinuation (cART persistence) and defined it broadly as ceasing cART or dropping out of active care. The 23 studies that found no significant association between depression and adherence and persistence to cART also tended to have a smaller sample size (mean sample size $=195$ ) as compared to the 35 studies that found positive associations (mean sample size $=477)$.
Some of the reasons for why the studies of depressive disorders found differing associations with adherence to cART were likely attributed to the differences in whether they utilized specific depression screening measures/ symptom rating scales (CES-D, BSI, PHQ) or standard diagnostic tools of depressive disorder (SCID, MINI, CIDI) to define the variable of "depressive disorder". Most of the studies that did not report a significant association with adherence utilized more standard diagnostic tools of depressive disorder such as the SCID, MINI, and CIDI. It would be important for future research evaluations of depression in HIV adherence research to improve data harmonization by utilizing the categorization of depression instruments that Simoni et al. [42] described as follows: [1] standardized diagnostic interviews, that are commonly used to assess the categorical diagnosis of depression based on DSM or ICD criteria, (including in this category CIDI, MINI, SCID, HADS, BDI etc.) [2] depression screening instruments, that provide empirically based cut-offs and are useful as the basis for referrals to more comprehensive evaluations or to estimate the prevalence of possible depression (including in this category CES-D and HSCL etc.), and [3] symptom-rating scales, that are useful for monitoring change in depression symptoms over time (including in this category BDI, CES-D, HSCL, MADRS etc.) [42]. Although there is no gold-standard for evaluation of depression, it has been recommended that researchers need to make informed choices based on the characteristics of the study population and the purpose of the research [42]. It is clear from this review that such rigorous evaluation of the study intentions needs to be applied in order to improve data harmonization efforts in the future.

Other causes of differences in results of the depression studies were likely due to the heterogeneity of the diagnostic and/or screening tools used for assessing depression. For instance the CES-D, identified as a standardized tool to screen for depressive symptoms, was the most commonly used instrument among the papers included in this review and it has twenty items, but not all versions are DSM-IV compatible (i.e., early versions do not include items that map directly onto DSM symptoms) [42]. Perhaps some of the variability in studies evaluating the effect of depression on cART adherence may have been associated with the different versions of the CES-D as well as the different cutoffs used to assess severity of depressive symptoms. Uniform assessment of depression is essential in order to improve efforts at data harmonization across studies of PLWHA. Harmonization of data can increase the likelihood of finding an association with a common outcome variable such as depression and adherence to cART. Furthermore, if the goal of the research study is to identify individuals with a clinical diagnosis of major depressive 
disorder, investigators should consider following up individuals who screen positive for depressive symptoms with a full diagnostic interview such as the SCID, MINI or CIDI, all of which have been validated with PLWHA in both developed and developing countries [42, 129]. Investigators should also be careful when using medical records (ICD-9 or DSM-IV codes) to identify participants as having a diagnosis of 'depression', as was done in a few of the evaluated studies. This particular method may misidentify a previous diagnosis of depression in a possibly successfully treated participant thereby affecting the analysis of the adherence outcome variable.

The majority of the studies ( $N=17 / 20$ articles) evaluating the impact of 'specific mental disorders other than depression' on cART adherence and persistence (see Table 2 in the electronic appendix) assessed anxiety disorders. Anxiety disorders were associated with decreased adherence in seven of the studies [unspecified anxiety disorder $(N=3)$, GAD $(N=1)$, PTSD $(N=2)$, panic disorder $(N=1)$ ]; but no association was detected in the majority of the studies $(N=15))$ [Unspecified anxiety disorder $(N=4)$, GAD $(N=2)$, PTSD $(N=6)$, panic disorder $(N=2)$, and agoraphobia $(N=1)$. Although two studies [unspecified anxiety disorder $(N=1)$, PTSD $(N=1)$ ] reported data suggesting that anxiety disorders were associated with increased adherence to cART, one study was conducted with a control group of participants with high levels of depression, rather than a typical control group without any mental disorder [110]; and the second study, concluded that "screening positive for any anxiety disorder reduced the risk of failing to take medications as directed" [89]. Although the gross sample size of these studies seems sufficient, the number of affected participants for each specific anxiety disorder may in effect not have been large enough to reach statistical significance. Given the high lifetime prevalence of anxiety disorders among PLWHA (7 \%) [130], however, studies evaluating individual anxiety disorders are not sufficient in number. To obtain a better understanding of the impact of specific mental disorders on cART adherence other than depression, future well-designed prospective cohort studies and RCTs with larger sample sizes for each specific disorder should be conducted to assess for causality.

All of the studies assessing the impact of 'unspecified mental disorders' on cART adherence concluded that mental disorders are associated with decreased adherence to cART. Importantly though, all of these studies included depression/depressive symptoms as the mental disorder variable [6, 113-117]. The result of these studies could therefore be attributed to the direct effect of the depressive disorder on adherence to cART. Only one study, which assessed cART persistence as the dependent variable, concluded that a severe mental disorder was significantly associated with lower probability for cART discontinuation in the first and second years (suggesting increased cART persistence), compared to participants with no mental disorders [120]. An additional finding of this study was that persons with a mental disorder and with six or more mental health visits per year were significantly less likely to discontinue cART compared to those with no mental health visits. It is therefore plausible that increased mental health visits in this study contributed to improved cART persistence by enhancing linkage to health care, including HIVassociated health-care [120].

The treatment of depression with antidepressants was found to be associated with improved adherence in seven of the nine studies (78\%) examining this effect. One of these studies had a very small sample size of only nine participants on ADT, therefore rendering any conclusions questionable [122]. Three of the four studies that found improved adherence to cART among participants adherent to ADT used pharmacy records to measure adherence [123, $125,127]$. The use of pharmacy records as the sole indicator of adherence could confound the adherence outcome as participants who obtain their antidepressant medications directly from pharmacies are more likely to pick up their cART medications, thus "hiding" the direct effect of ADT on cART adherence (via the improvement of depressive symptoms). Furthermore, adherence to one medication may indicate a propensity towards adherence in general. Therefore participants who are adherent to ADT may be more likely to adhere to cART simply because they are more adherent as a rule, and not because of improved depressive symptoms. In one study that did not find an association between ADT and cART adherence, starting psychiatric treatment was broadly defined as receiving a diagnosis of depression, seeing a psychiatrist or taking ADT, therefore making the individual effects of pharmacological, and non-pharmacological psychiatric treatment impossible to unfurl [11]. A recent RCT of $137 \mathrm{HIV}+$ homeless and marginally housed persons found no significant difference in cART adherence among those receiving directly observed fluoxetine as compared to the those referred to the community for psychiatric care [128]. This study however had high levels of persistence to cART at the end of study in both groups (73 vs $75 \%$ NS), as well as similar percentages of viral suppression in both groups (specific data not reported). These results suggest that perhaps persistence to cART may be more significant than adherence for a durable cART regimen.

Issues regarding differences between the studies in specific ADTs as well as appropriate individual dosing, however, make interpretation of the impact of ADT on cART adherence for depression difficult. Little evidence from randomized controlled clinical trials is available to guide the psychiatric treatment of PLWHA [131]. Some 
studies, however, have shown that depression can be effectively treated in PLWHA [132-134], specifically using selective serotonin reuptake inhibitors (SSRIs) [135, 136]. Despite some possible evidence based from this review that ADT may improve adherence to cART among PLWHA who have co-morbid depression, it is still unclear at this time whether treating depressive disorders with ADT improves adherence to cART. Although other ADT besides SSRIs including citalopram [137] and tricyclic antidepressants [136, 138], have been shown to be effective in treating depression among PLWHA, SSRIs [136, 139] are better tolerated in PLWHA and recommended as firstline agents for depression [140]. In devising research involving antidepressants for PLWHA, however, clinicians should control for the stage of HIV illness, co-morbid illnesses such as hepatitis B and C, the potential for drug interactions with cART, type of ADT, maximal dosing and participants' preferences [133, 141-143].

This systematic review also identified a large heterogeneity of measurements and definitions of adherence to cART in various studies likely contributing to the variation in outcomes. Overall the majority of studies utilized some form of self-report, while fewer utilized EDMS, pharmacy refill reviews, and unannounced pill counts. There is no universally accepted 'gold' standard for the measurement of cART adherence [144]. Self-report adherence measures and other indirect measures such as EDMs and pill counts have been found to distinguish between clinically meaningful patterns of medication-taking behavior [145, 146]. Self-report, however, tends to overestimate adherence by 10-20\% as compared to EDMs evaluations because selfreport is susceptible to recall bias, inaccurate memory and potentially to social desirability bias [147-149]. Pharmacy record review, on the other hand, has been show to misclassify participants as non-adherent to cART in up to $43 \%$ of the time when disregarding leftover medication [150]. The variations in measurements utilized to evaluate adherence to cART in the reviewed studies, as well as the definition of recall period, likely contributed to the great variation of the primary outcome, cART adherence.

There are some limitations to this review. Although we were very rigorous with inclusion criteria and exclusion criteria, it is plausible that some relevant papers that should have been included were missed. Additionally, the exclusion of behavioral treatments for depression and other mental disorders constitutes a limitation when discussing the scope of mental disorder treatments. Behavioral treatments have overall been found to be effective in the treatment of mental disorders, but due to the length of the paper and the large quantity of data to be processed in the scope of pharmacological treatment alone, it was decided to not include studies on behavioral treatments in this particular review. The inclusion of studies from both developed and developing countries also could have introduced bias into our findings since confounding factors such as barriers and facilitators of cART adherence may be specific to different geographic areas [151-153]. In developing countries, food insufficiency [154-156] and health system deficiencies have been found to undermine treatment continuity and adherence to cART [157], whereas religiosity has been shown to be positively correlated with cART adherence [158]. Conversely, examining data from different parts of the world increases the generalizability of our findings and emphasizes the widespread nature of both mental disorders and HIV.

Despite the limitations of the review, this is the first paper to exhaustively review studies evaluating the association between DSM-IV mental disorders (excluding substance use disorders) and adherence and persistence to cART among adult PLWHA. Most of the studies included in this review studied the adherence variable at a time period when cART was more complex, requiring multidose regimens. Current preferred once-daily dosing regimens, however, have been recently shown to improve adherence [159-162]. In a time when cART complexity has decreased while potency of regimens has increased, persistence to cART may be a superior indicator of HIV outcomes than cART adherence and a better variable to evaluate $[4,159,163,164]$. Recent studies have found data suggesting that adequate cART adherence may not be the key factor in viral suppression [165]. Future studies should evaluate the impact of mental disorders and their treatment on HIV outcomes other than adherence such as maximal viral suppression (i.e. HIV VL $<48$ copies $/ \mathrm{mL}$ ), neurocognitive deficits, as well as liver and renal dysfunction.

\section{Conclusions}

The majority of the studies included in this systematic review identified a significant association between depressive symptoms/disorder and cART nonadherence and non-persistence. Data related to the impact of specific mental disorders other than depression (anxiety disorders, bipolar disorder, psychotic disorders and personality disorders) on adherence to cART are insufficient and inconsistent. Future research should focus specifically on each of these mental disorders and cART adherence. Additionally, common measures should be used to assess cART adherence as well as specific mental disorders to improve data harmonization across studies. Furthermore, the majority of existing data suggest that treatment of depression with ADT may be associated with improved adherence to cART among PLWHA. Future RCT-designed studies will need to be conducted to best determine the association between improvement of depressive symptomatology and cART 
adherence. Lastly, HIV outcomes other than adherence to cART, such as maximal HIV viral load suppression and neurocognitive deficits are important to evaluate now that more potent combination antiretroviral regimens are available.

Acknowledgments Angela DiPaola for her assistance in proofreading this manuscript. Funding is provided by the National Institutes on Drug Abuse (K02-DA032322) for SAS

Open Access This article is distributed under the terms of the Creative Commons Attribution License which permits any use, distribution, and reproduction in any medium, provided the original author(s) and the source are credited.

\section{References}

1. Crum NF, Riffenburgh RH, Wegner S, et al. Comparisons of causes of death and mortality rates among HIV-infected persons: analysis of the pre-, early, and late HAART (highly active antiretroviral therapy) eras. J Acquir Immune Defic Syndr. 2006; 41(2):194-200.

2. Bhaskaran K, Hamouda O, Sannes M, et al. Changes in the risk of death after HIV seroconversion compared with mortality in the general population. JAMA. 2008;300(1):51-9.

3. Lima VD, Harrigan R, Bangsberg DR, et al. The combined effect of modern highly active antiretroviral therapy regimens and adherence on mortality over time. J Acquir Immune Defic Syndr. 2009;50(5):529-36.

4. Bae JW, Guyer W, Grimm K, Altice FL. Medication persistence in the treatment of HIV infection: a review of the literature and implications for future clinical care and research. AIDS. 2011; 25(3):279-90.

5. de Bruin M, Hospers HJ, van Breukelen GJ, Kok G, Koevoets WM, Prins JM. Electronic monitoring-based counseling to enhance adherence among HIV-infected patients: a randomized controlled trial. Health Psychol. 2010;29(4):421-8.

6. Paterson DL, Swindells S, Mohr J, et al. Adherence to protease inhibitor therapy and outcomes in patients with HIV infection. Ann Intern Med. 2000;133(1):21-30.

7. Maggiolo F, Airoldi M, Kleinloog HD, et al. Effect of adherence to HAART on virologic outcome and on the selection of resistance-conferring mutations in NNRTI- or PI-treated patients. HIV Clin Trials. 2007;8(5):282-92.

8. Harrigan PR, Hogg RS, Dong WW, et al. Predictors of HIV drug-resistance mutations in a large antiretroviral-naive cohort initiating triple antiretroviral therapy. J Infect Dis. 2005;191(3): 339-47.

9. Bouhnik AD, Preau M, Vincent E, et al. Depression and clinical progression in HIV-infected drug users treated with highly active antiretroviral therapy. Antivir Ther. 2005;10(1):53-61.

10. Escobar I, Campo M, Martín J, Fernández-Shaw C, Pulido F, Rubio R. Factors affecting patient adherence to highly active antiretroviral therapy. Ann Pharmacother. 2003;37(6):775-81.

11. Glass TR, Battegay M, Cavassini M, et al. Longitudinal analysis of patterns and predictors of changes in self-reported adherence to antiretroviral therapy: Swiss HIV Cohort Study. J Acquir Immune Defic Syndr. 2010;54(2):197-203.

12. Halkitis P, Palamar J, Mukherjee P. Analysis of HIV medication adherence in relation to person and treatment characteristics using hierarchical linear modeling. AIDS Patient Care STDs. 2008;22(4):323-35.

13. Azar MM, Springer SA, Meyer JP, Altice FL. A systematic review of the impact of alcohol use disorders on HIV treatment outcomes, adherence to antiretroviral therapy and health care utilization. Drug Alcohol Depend. 2010;112(3):178-93.

14. Arnsten JH, Li X, Mizuno Y. Factors associated with antiretroviral therapy adherence and medication errors among HIVinfected injection drug users. J Acquir Immune Defic Syndr. 2007;46(Suppl 2):S64-71.

15. Duggan JM, Locher A, Fink B, Okonta C, Chakraborty J. Adherence to antiretroviral therapy: a survey of factors associated with medication usage. AIDS Care. 2009;21(9):1141-7.

16. Li X, Huang L, Wang H, Fennie KP, He G, Williams AB. Stigma mediates the relationship between self-efficacy, medication adherence, and quality of life among people living with HIV/AIDS in China. AIDS Patient Care STDs. 2011;25(11): 665-71.

17. Van Tam V, Pharris A, Thorson A, Alfven T, Larsson M. "It is not that I forget, it's just that I don't want other people to know": barriers to and strategies for adherence to antiretroviral therapy among HIV patients in Northern Vietnam. AIDS Care. 2011;23(2):139-45.

18. Anand P, Springer SA, Copenhaver MM, Altice FL. Neurocognitive impairment and HIV risk factors: a reciprocal relationship. AIDS Behav. 2010;14(6):1213-26.

19. Tegger MK, Crane HM, Tapia KA, Uldall KK, Holte SE, Kitahata MM. The effect of mental illness, substance use, and treatment for depression on the initiation of highly active antiretroviral therapy among $\mathrm{HIV}$-infected individuals. AIDS Patient Care STDS. 2008;22(3):233-43.

20. DeLorenze GN, Satre DD, Quesenberry CP, Tsai AL, Weisner CM. Mortality after diagnosis of psychiatric disorders and co-occurring substance use disorders among HIV-infected patients. AIDS Patient Care STDs. 2010;24(11):705-12.

21. American Psychiatric Association. Diagnostic and statistical manual of mental disorders. 4th ed., text revision. Washington, DC: Author; 2000.

22. Kessler C. Prevalence and Treatment of Mental Disorders, 1990 to 2003. NEJM. 2005;352:2515-23.

23. Lopes M, Olfson M, Rabkin J, et al. Gender, HIV status, and psychiatric disorders: results from the National Epidemiologic Survey on Alcohol and Related Conditions. J Clin Psychiatry. 2011;73(3):384-91.

24. Whetten K, Reif SS, Napravnik S, et al. Substance abuse and symptoms of mental illness among HIV-positive persons in the Southeast. South Med J. 2005;98(1):9-14.

25. Bing EG, Burnam MA, Longshore D, et al. Psychiatric disorders and drug use among human immunodeficiency virus-infected adults in the United States. Arch Gen Psychiatry. 2001;58(8): 721-8.

26. Kessler RC, Berglund P, Demler O, et al. The epidemiology of major depressive disorder: results from the National Comorbidity Survey Replication (NCS-R). JAMA. 2003;289(23): 3095-105.

27. Blazer DG, Kessler RC, McGonagle KA, Swartz MS. The prevalence and distribution of major depression in a national community sample: the National Comorbidity Survey. Am J Psychiatry. 1994;151(7):979-86.

28. Orlando M, Burnam MA, Beckman R, et al. Re-estimating the prevalence of psychiatric disorders in a nationally representative sample of persons receiving care for HIV: results from the HIV Cost and Services Utilization Study. Int J Methods Psychiatr Res. 2002;11(2):75-82.

29. Buathong N, Hiransuthikula N, Tangwongchaib S, Komoltric C. Association between depression and adherence to highly active 
antiretroviral therapy among adult HIV infected patients in Thailand. Asian Biomedicine. 2009;3(2):127-33.

30. Etienne M, Hossain M, Redfield R, Stafford K, Amoroso A. Indicators of adherence to antiretroviral therapy treatment among HIV/AIDS patients in 5 African countries. J Int Assoc Physicians AIDS Care. 2010;9(2):98-103.

31. Carrico AW, Bangsberg DR, Weiser SD, Chartier M, Dilworth SE, Riley ED. Psychiatric correlates of HAART utilization and viral load among HIV-positive impoverished persons. AIDS. 2011;25(8):1113-8.

32. Lima VD, Geller J, Bangsberg DR, et al. The effect of adherence on the association between depressive symptoms and mortality among HIV-infected individuals first initiating HAART. AIDS. 2007;21(9):1175-83.

33. Boarts JM, Buckley-Fischer BA, Armelie AP, Bogart LM, Delahanty DL. The impact of HIV diagnosis-related vs. nondiagnosis related trauma on PTSD, depression, medication adherence, and HIV disease markers. J Evid Based. Soc Work. 2009;6(1):4-16.

34. Keuroghlian AS, Kamen CS, Neri E, Lee S, Liu R, Gore-Felton C. Trauma, dissociation, and antiretroviral adherence among persons living with HIV/AIDS. J Psychiatr Res. 2011;45(7):942-8.

35. Moore DJ, Posada C, Parikh M, et al. The HIV Neurobehavioral Research Program (HNRP). HIV-infected individuals with cooccurring bipolar disorder evidence poor antiretroviral and psychiatric medication adherence. AIDS Behav. 2011. doi: 10.1007/s10461-011-0072-2.

36. Mellins CA, Havens JF, McDonnell C, et al. Adherence to antiretroviral medications and medical care in HIV-infected adults diagnosed with mental and substance abuse disorders. AIDS Care. 2009;21(2):168-77.

37. Gonzalez JS, Batchelder AW, Psaros C, Safren SA. Depression and HIV/AIDS treatment nonadherence: a review and metaanalysis. J Acquir Immune Defic Syndr. 2011;58(2):181-7.

38. Campos LN, Guimaraes MD, Remien RH. Anxiety and depression symptoms as risk factors for non-adherence to antiretroviral therapy in Brazil. AIDS Behav. 2010;14(2):289-99.

39. Liberati A, Altman DG, Tetzlaff J, et al. The PRISMA statement for reporting systematic reviews and meta-analyses of studies that evaluate health care interventions: explanation and elaboration. J Clin Epidemiol. 2009;62(10):e1-34.

40. World Health Organization. International statistical classification of diseases and related health problems, 10th Revision (ICD-10). 2007. http://www.who.int/classifications/icd/en/.

41. CDC. International classification of diseases, ninth revision, clinical modification (ICD-9-CM). http://www.cdc.gov/nchs/ icd $/$ icd $9 \mathrm{~cm} . h \mathrm{htm}$.

42. Simoni JM, Safren SA, Manhart LE, et al. Challenges in addressing depression in HIV research: assessment, cultural context, and methods. AIDS Behav. 2011;15(2):376-88.

43. Cramer JA, Roy A, Burrell A, et al. Medication compliance and persistence: terminology and definitions. Value Health. 2008;11(1): 44-7.

44. Landis JR, Koch GG. The measurement of observer agreement for categorical data. Biometrics. 1977;33(1):159-74.

45. Beekman ATF, Deeg DJH, Van Limbeek J, Braam AW, et al. Criterion validity of the Center for Epidemiologic Studies Depression scale (CES-D): results from a community-based sample of older subjects in the Netherlands. Psychol Med. 1997; 27(1):231-5.

46. Radloff LS. The CES-D scale: a self-report depression scale for research in the general population. Appl Psychol Meas. 1977;1: 385-401.

47. Beck AT, Steer RA, Carbin MG. Psychometric properties of the Beck Depression Inventory: twenty-five years of evaluation. Clin Psychol Rev. 1988;8(1):77-100.
48. Beck A, Ward C, Mendelson M, Mock J, Erbaugh J. An inventory for measuring depression. Arch Gen Psychiatry. 1961;4:561-71.

49. Beck A. Beck depression inventory-II. San Antonio: Harcourt Brace and Company/The Psychological Corporation; 1996.

50. Lipps GE, Lowe GA, De La Haye W, et al. Validation of the Beck Depression Inventory II in HIV-positive patients. West Indian Med J. 2010;59(4):374-9.

51. First MB, Spitzer R, Gibbon M, Williams JBW. Structured Clinical Interview for DSM-IV Axis I disorders, Clinician Version (SCID-CV). Washington: American Psychiatric Press; 1996.

52. Montgomery SA, Asberg M. A new depression scale designed to be sensitive to change. Br J Psychiatry. 1979;134:382-9.

53. Derogatis LR, Melisaratos N. The Brief Symptom Inventory: an introductory report. Psychol Med. 1983;13(03):595-605.

54. Rogers WH, Wilson IB, Bungay KM, Cynn DJ, Adler DA. Assessing the performance of a new depression screener for primary care (PC-SAD). J Clin Epidemiol. 2002;55(2):164-75.

55. Mitchell AJ, Bird V, Rizzo M, Meader N. Diagnostic validity and added value of the Geriatric Depression Scale for depression in primary care: a meta-analysis of GDS30 and GDS15. J Affect Disord. 2010;125(1-3):10-7.

56. Hyphantis T, Kotsis K, Voulgari PV, Tsifetaki N, Creed F, Drosos AA. Diagnostic accuracy, internal consistency, and convergent validity of the Greek version of the patient health questionnaire 9 in diagnosing depression in rheumatologic disorders. Arthritis Care Res. 2011;63(9):1313-21.

57. Etienne M, Hossain M, Redfield R, Stafford K, Amoroso A. Indicators of adherence to antiretroviral therapy treatment among HIV/AIDS patients in 5 African countries. J Int Assoc Physicians AIDS Care (Chic Ill). 2010;9(2):98-103.

58. Mohammed H, Kieltyka L, Richardson-Alston G, et al. Adherence to HAART among HIV-infected persons in rural Louisiana. AIDS Patient Care STDS. 2004;18(5):289-96.

59. Boarts JM, Sledjeski EM, Bogart LM, Delahanty DL. The differential impact of PTSD and depression on HIV disease markers and adherence to HAART in people living with HIV. AIDS Behav. 2006;10(3):253-61.

60. Carrieri MP, Chesney MA, Spire B, et al. Failure to maintain adherence to HAART in a cohort of French HIV-positive injecting drug users. Int J Behav Med. 2003;10(1):1-14.

61. Carrieri MP, Leport C, Protopopescu C, et al. Factors associated with nonadherence to highly active antiretroviral therapy: a 5-year follow-up analysis with correction for the bias induced by missing data in the treatment maintenance phase. J Acquir Immune Defic Syndr. 2006;41(4):477-85.

62. Kacanek D, Jacobson DL, Spiegelman D, Wanke C, Isaac R, Wilson IB. Incident depression symptoms are associated with poorer HAART adherence: a longitudinal analysis from the Nutrition for Healthy Living study. J Acquir Immune Defic Syndr. 2010;53(2):266-72.

63. Kleeberger CA, Buechner J, Palella F, et al. Changes in adherence to highly active antiretroviral therapy medications in the Multicenter AIDS Cohort Study. AIDS. 2004;18(4):683-8.

64. Lazo M, Gange SJ, Wilson TE, et al. Patterns and predictors of changes in adherence to highly active antiretroviral therapy: longitudinal study of men and women. Clin Infect Dis. 2007; 45(10): 1377-85.

65. Protopopescu C, Raffi F, Roux P, et al. Factors associated with non-adherence to long-term highly active antiretroviral therapy: a 10 year follow-up analysis with correction for the bias induced by missing data. JAC. 2009;64(3):599-606.

66. Singh N, Squier C, Sivek C, Wagener M, Hong Nguyen M, Yu VL. Determinants of compliance with antiretroviral therapy in patients with human immunodeficiency virus: prospective 
assessment with implications for enhancing compliance. AIDS Care. 1996;8(3):261-9.

67. Spire B, Duran S, Souville M, Leport C, Raffi F, Moatti JP. Adherence to highly active antiretroviral therapies (HAART) in HIV-infected patients: from a predictive to a dynamic approach. Soc Sci Med. 2002;54(10):1481-96.

68. Tucker JS, Burnam MA, Sherbourne CD, Kung FY, Gifford AL. Substance use and mental health correlates of nonadherence to antiretroviral medications in a sample of patients with human immunodeficiency virus infection. Am J Med. 2003;114(7): 573-80.

69. Cruess DG, Kalichman SC, Amaral C, Swetzes C, Cherry C, Kalichman MO. Benefits of adherence to psychotropic medications on depressive symptoms and antiretroviral medication adherence among men and women living with HIV/AIDS. Ann Behav Med. 2011;43(2):189-97.

70. Gonzalez JS, Psaros C, Batchelder A, Applebaum A, Newville $\mathrm{H}$, Safren SA. Clinician-assessed depression and HAART adherence in HIV-infected individuals in methadone maintenance treatment. Ann Behav Med. 2011;42(1):120-6.

71. Rodkjaer L, Laursen T, Christensen NB, Lomborg K, Ostergaard L, Sodemann M. Changes in depression in a cohort of Danish HIV-positive individuals: time for routine screening. Sex Health. 2011;8(2):214-21.

72. Vranceanu AM, Safren SA, Lu M, et al. The relationship of post-traumatic stress disorder and depression to antiretroviral medication adherence in persons with HIV. AIDS Patient Care STDS. 2008;22(4):313-21.

73. Arnsten JH, Li X, Mizuno Y, et al. Factors associated with antiretroviral therapy adherence and medication errors among HIV-infected injection drug users. J Acquir Immune Defic Syndr. 2007;46(Suppl. 2):S64-71.

74. Diiorio C, McCarty F, Depadilla L, et al. Adherence to antiretroviral medication regimens: a test of a psychosocial model. AIDS Behav. 2009;13(1):10-22.

75. Do NT, Phiri K, Bussmann H, Gaolathe T, Marlink RG, Wester CW. Psychosocial factors affecting medication adherence among HIV-1 infected adults receiving combination antiretroviral therapy (cART) in Botswana. AIDS Res Hum Retroviruses. 2010;26(6):685-91.

76. Olisah VO, Baiyewu O, Sheikh TL. Adherence to highly active antiretroviral therapy in depressed patients with HIV/AIDS attendinga Nigerian university teaching hospital clinic. Afr J Psychiatry (Johannesbg). 2010;13(4):275-9.

77. Phillips KD, Moneyham L, Murdaugh C, et al. Sleep disturbance and depression as barriers to adherence. Clin Nurs Res. 2005; 14(3):273-93.

78. Rodkjaer L, Laursen T, Balle N, Sodemann M. Depression in patients with HIV is under-diagnosed: a cross-sectional study in Denmark. HIV Med. 2010;11(1):46-53.

79. Royal SW, Kidder DP, Patrabansh S, et al. Factors associated with adherence to highly active antiretroviral therapy in homeless or unstably housed adults living with HIV. AIDS Care. 2009;21(4):448-55.

80. Sarna A, Pujari S, Sengar AK, Garg R, Gupta I, Dam J. Adherence to antiretroviral therapy \& its determinants amongst HIV patients in India. Indian J Med Res. 2008;127(1):28-36.

81. Schuman P. Prescription of and adherence to antiretroviral therapy among women with AIDS. AIDS Behav. 2001;5(4): 371-8.

82. Tadios Y, Davey G. Antiretroviral treatment adherence and its correlates in Addis Ababa, Ethiopia. Ethiop Med J. 2006;44(3): 237-44.

83. Waldrop-Valverde D, Valverde E. Homelessness and psychological distress as contributors to antiretroviral nonadherence in
HIV-positive injecting drug users. AIDS Patient Care STDS. 2005;19(5):326-34

84. Li L, Lee SJ, Wen Y, Lin C, Wan D, Jiraphongsa C. Antiretroviral therapy adherence among patients living with HIV/AIDS in Thailand. Nurs Health Sci. 2010;12(2):212-20.

85. Rao D, Feldman BJ, Fredericksen RJ, et al. A structural equation model of HIV-related stigma, depressive symptoms, and medication adherence. AIDS Behav. 2011;16:711-6.

86. Wagner GJ, Goggin K, Remien RH, et al. A closer look at depression and its relationship to HIV antiretroviral adherence. Ann Behav Med. 2011;42(3):352-60.

87. Berger-Greenstein JA, Cuevas CA, Brady SM, Trezza G, Richardson MA, Keane TM. Major depression in patients with HIV/AIDS and substance abuse. AIDS Patient Care STDS. 2007; 21(12):942-55.

88. Catz SL, Kelly JA, Bogart LM, Benotsch EG, McAuliffe TL. Patterns, correlates, and barriers to medication adherence among persons prescribed new treatments for HIV disease. Health Psychol. 2000;19(2):124-33.

89. Gonzalez JS, Penedo FJ, Antoni MH, et al. Social support, positive states of mind, and HIV treatment adherence in men and women living with HIV/AIDS. Health Psychol. 2004;23(4):413-8.

90. Gordillo V, del Amo J, Soriano V, Gonzalez-Lahoz J. Sociodemographic and psychological variables influencing adherence to antiretroviral therapy. AIDS. 1999;13(13):1763-9.

91. Ingersoll $\mathrm{K}$. The impact of psychiatric symptoms, drug use, and medication regimen on non-adherence to HIV treatment. AIDS Care. 2004;16(2):199-211.

92. Leserman J, Ironson G, O’Cleirigh C, Fordiani JM, Balbin E. Stressful life events and adherence in HIV. AIDS Patient Care STDS. 2008;22(5):403-11.

93. Mugavero M, Ostermann J, Whetten K, et al. Barriers to antiretroviral adherence: the importance of depression, abuse, and other traumatic events. AIDS Patient Care STDS. 2006;20(6): 418-28.

94. Palmer NB, Salcedo J, Miller AL, Winiarski M, Arno P. Psychiatric and social barriers to HIV medication adherence in a triply diagnosed methadone population. AIDS Patient Care STDS. 2003;17(12):635-44.

95. Shin S, Munoz M, Espiritu B, et al. Psychosocial impact of poverty on antiretroviral nonadherence among HIV-TB coinfected patients in Lima, Peru. J Int Assoc Physicians AIDS Care (Chic Ill). 2008;7(2):74-81.

96. van Servellen G, Chang B, Garcia L, Lombardi E. Individual and system level factors associated with treatment nonadherence in human immunodeficiency virus-infected men and women. AIDS Patient Care STDS. 2002;16(6):269-81.

97. Johnson MO, Dilworth SE, Taylor JM, Darbes LA, Comfort ML, Neilands TB. Primary relationships, HIV treatment adherence, and virologic control. AIDS Behav. 2011. doi:10.1007/ s10461-011-0021-0.

98. Kyser M, Buchacz K, Bush TJ, et al. Factors associated with non-adherence to antiretroviral therapy in the SUN study. AIDS Care. 2011;23(5):601-11.

99. Bottonari KA, Safren SA, McQuaid JR, Hsiao CB, Roberts JE. A longitudinal investigation of the impact of life stress on HIV treatment adherence. J Behav Med. 2010;33(6):486-95.

100. Gibbie T, Hay M, Hutchison CW, Mijch A. Depression, social support and adherence to highly active antiretroviral therapy in people living with HIV/AIDS. Sex Health. 2007;4(4):227-32.

101. Moss AR, Hahn JA, Perry S, et al. Adherence to highly active antiretroviral therapy in the homeless population in San Francisco: a prospective study. Clin Infect Dis. 2004;39(8):1190-8.

102. Kalichman SC, Pellowski J, Kalichman MO, et al. Food insufficiency and medication adherence among people living with 
HIV/AIDS in urban and peri-urban settings. Prev Sci. 2011;12(3): 324-32.

103. Wagner GJ, Bogart LM, Galvan FH, Banks D, Klein DJ. Discrimination as a key mediator of the relationship between posttraumatic stress and HIV treatment adherence among African American men. J Behav Med. 2011;35(1):8-18.

104. Ammassari A, Antinori A, Aloisi MS. Depressive symptoms, neurocognitive impairment, and adherence to highly active antiretroviral therapy among HIV-infected persons. Psychosomatics. 2004;45(5):394-402.

105. Bianco JA, Heckman TG, Sutton M, Watakakosol R, Lovejoy T. Predicting adherence to antiretroviral therapy in HIV-infected older adults: the moderating role of gender. AIDS Behav. 2011;15(7):1437-46.

106. Farley J, Miller E, Zamani A, et al. Screening for hazardous alcohol use and depressive symptomatology among HIV-infected patients in Nigeria: prevalence, predictors, and association with adherence. J Int Assoc Physicians AIDS Care (Chic Ill). 2010;9(4):218-26.

107. Herrmann S, McKinnon E, John M, et al. Evidence-based, multifactorial approach to addressing non-adherence to antiretroviral therapy and improving standards of care. Intern Med J. 2008;38(1):8-15.

108. Springer SA, Chen S, Altice F. Depression and symptomatic response among HIV-infected drug users enrolled in a randomized controlled trial of directly administered antiretroviral therapy. AIDS Care. 2009;21(8):976-83.

109. Catz SL, Heckman TG, Kochman A, DiMarco M. Rates and correlates of HIV treatment adherence among late middle-aged and older adults living with HIV disease. Health Psychol. 2001;6(1):47-58.

110. Sledjeski EM, Delahanty DL, Bogart LM. Incidence and impact of posttraumatic stress disorder and comorbid depression on adherence to HAART and CD4+ counts in people living with HIV. AIDS Patient Care STDs. 2005;19(11):728-36.

111. Nilsson Schönnesson L, Williams ML, Ross MW, Bratt G, Keel B. Factors associated with suboptimal antiretroviral therapy adherence to dose, schedule, and dietary instructions. AIDS Behav. 2007;11(2):175-83.

112. Wagner GJ, Kanouse DE, Koegel P, Sullivan G. Adherence to HIV antiretrovirals among persons with serious mental illness. AIDS Patient Care STDS. 2003;17(4):179-86.

113. Grierson J, Koelmeyer RL, Smith A, Pitts M. Adherence to antiretroviral therapy: factors independently associated with reported difficulty taking antiretroviral therapy in a national sample of HIV-positive Australians. HIV Med. 2011;12(9): $562-9$.

114. Adewuya AO, Afolabi MO, Ola BA, et al. The effect of psychological distress on medication adherence in persons with HIV infection in Nigeria. Psychosomatics. 2010;51(1):68-73.

115. Kumar V, Encinosa W. Effects of HIV medication complexity and depression on adherence to HIV medication. Patient. 2010; 3(1):59-69.

116. Soto Blanco JM, Ruiz Pérez I, De Labry Lima AO, Castro Recio JM, Girela López E, Antón Basanta JJ. Adherence to antiretroviral treatment in prisons. AIDS Res Hum Retroviruses. 2005;21(8):683-8.

117. Mellins CA, Kang E, Leu CS, Havens JF, Chesney MA. Longitudinal study of mental health and psychosocial predictors of medical treatment adherence in mothers living with HIV disease. AIDS Patient Care STDS. 2003;17(8):407-16.

118. Li X, Margolick JB, Conover CS, et al. Interruption and discontinuation of highly active antiretroviral therapy in the multicenter AIDS cohort study. J Acquir Immune Defic Syndr. 2005;38(3):320-8.
119. Maru DS, Bruce RD, Walton M, et al. Initiation, adherence, and retention in a randomized controlled trial of directly administered antiretroviral therapy. AIDS Behav. 2008;12(2):284-93.

120. Himelhoch S, Brown $\mathrm{CH}$, Walkup J, et al. HIV patients with psychiatric disorders are less likely to discontinue HAART. AIDS. 2009;23(13):1735-42.

121. Walkup JT, Sambamoorthi U, Crystal S. Use of newer antiretroviral treatments among HIV-infected medicaid beneficiaries with serious mental illness. J Clin Psychiatry. 2004;65(9):1180-9.

122. Dalessandro M, Conti CM, Gambi F, et al. Antidepressant therapy can improve adherence to antiretroviral regimens among HIV-infected and depressed patients. J Clin Psychopharmacol. 2007;27(1):58-61.

123. Horberg MA, Silverberg MJ, Hurley LB, et al. Effects of depression and selective serotonin reuptake inhibitor use on adherence to highly active antiretroviral therapy and on clinical outcomes in HIV-infected patients. J Acquir Immune Defic Syndr. 2008;47(3):384-90.

124. Kumar V, Encinosa W. Effects of antidepressant treatment on antiretroviral regimen adherence among depressed HIV-infected patients. Psychiatr Q. 2009;80(3):131-41.

125. Yun LW, Maravi M, Kobayashi JS, Barton PL, Davidson AJ. Antidepressant treatment improves adherence to antiretroviral therapy among depressed HIV-infected patients. J Acquir Immune Defic Syndr. 2005;38(4):432-8.

126. Tsai AC, Weiser SD, Petersen ML, Ragland K, Kushel MB, Bangsberg DR. A marginal structural model to estimate the causal effect of antidepressant medication treatment on viral suppression among homeless and marginally housed persons with HIV. Arch Gen Psychiatry. 2010;67(12):1282-90.

127. Akincigil A, Wilson IB, Walkup JT, Siegel MJ, Huang C, Crystal S. Antidepressant treatment and adherence to antiretroviral medications among privately insured persons with HIV/ AIDS. AIDS Behav. 2011;15(8):1819-28.

128. Tsai AC, Karasic D, Hammer G, Charlebois E, Ragland K, Moss A, Sorenson J, Dilley J, Bangsberg D. Directly observed antidepressant medication treatment and HIV outcomes among homeless and marginally housed HIV+ adults: a randomized controlled trial. Am J Public Health. 2012;102(8), in press.

129. Maj M, Janssen R, Starace F, et al. WHO Neuropsychiatric AIDS study, cross-sectional phase I. Study design and psychiatric findings. Arch Gen Psychiatry. 1994;51(1):39-49.

130. Perkins DO, Stern RA, Golden RN, Murphy C, Naftolowitz D, Evans DL. Mood disorders in HIV infection: prevalence and risk factors in a nonepicenter of the AIDS epidemic. Am J Psychiatry. 1994;151(2):233-6.

131. Freudenreich O, Goforth HW, Cozza KL, et al. Psychiatric treatment of persons with HIV/AIDS: an HIV-psychiatry consensus survey of current practices. Psychosomatics. 2010;51(6): $480-8$.

132. Olatunji BO, Mimiaga MJ, O'Cleirigh C, Safren SA. Review of treatment studies of depression in HIV. Top HIV Med. 2006; 14(3):112-24.

133. Ferrando SJ, Freyberg Z. Treatment of depression in HIV positive individuals: a critical review. Int Rev Psychiatry. 2008; 20(1):61-71.

134. Rabkin JG. HIV and depression: 2008 review and update. Curr HIV/AIDS Rep. 2008;5(4):163-71.

135. Rabkin JG, Wagner G, Rabkin R. Effects of sertraline on mood and immune status in patients with major depression and HIV illness: an open trial. J Clin Psychiatry. 1994;55(10):433-9.

136. Elliott AJ, Uldall KK, Bergam K, Russo J, Claypoole K, RoyByrne PP. Randomized, placebo-controlled trial of paroxetine versus imipramine in depressed HIV-positive outpatients. Am J Psychiatry. 1998;155(3):367-72. 
137. Currier MB, Molina G, Kato M. Citalopram treatment of major depressive disorder in Hispanic HIV and AIDS patients: a prospective study. Psychosomatics. 2004;45(3):210-6.

138. Schwartz JA, McDaniel JS. Double-blind comparison of fluoxetine and desipramine in the treatment of depressed women with advanced HIV disease: a pilot study. Depress Anxiety. 1999; 9(2):70-4.

139. Ferrando SJ, Goldman JD, Charness WE. Selective serotonin reuptake inhibitor treatment of depression in symptomatic HIV infection and AIDS. Improvements in affective and somatic symptoms. Gen Hosp Psychiatry. 1997;19(2):89-97.

140. Elliott AJ, Roy-Byrne PP. Major Depressive Disorder and HIV1 Infection: a review of treatment trials. Semin Clin Neuropsychiatry. 1998;3(2):137-50.

141. Tseng AL, Foisy MM. Significant interactions with new antiretrovirals and psychotropic drugs. Ann Pharmacother. 1999;33(4): 461-73.

142. Wagner GJ, Maguen S, Rabkin JG. Ethnic differences in response to fluoxetine in a controlled trial with depressed HIVpositive patients. Psychiatr Serv. 1998;49(2):239-40.

143. Wilder CM, Elbogen EB, Moser LL, Swanson JW, Swartz MS. Medication preferences and adherence among individuals with severe mental illness and psychiatric advance directives. Psychiatr Serv. 2010;61(4):380-5.

144. Paterson DL, Potoski B, Capitano B. Measurement of adherence to antiretroviral medications. J Acquir Immune Defic Syndr. 2002;31(Suppl 3):S103-6.

145. Simoni JM, Kurth AE, Pearson CR, Pantalone DW, Merrill JO, Frick PA. Self-report measures of antiretroviral therapy adherence: a review with recommendations for HIV research and clinical management. AIDS Behav. 2006;10(3):227-45.

146. Nieuwkerk PT, Oort FJ. Self-reported adherence to antiretroviral therapy for HIV-1 infection and virologic treatment response: a meta-analysis. J Acquir Immune Defic Syndr. 2005;38(4): 445-8.

147. Arnsten JH, Demas PA, Farzadegan H, et al. Antiretroviral therapy adherence and viral suppression in HIV-infected drug users: comparison of self-report and electronic monitoring. Clin Infect Dis. 2001;33(8):1417-23.

148. Wagner G, Miller LG. Is the influence of social desirability on patients' self-reported adherence overrated? J Acquir Immune Defic Syndr. 2004;35(2):203-4.

149. Jonsdottir H, Opjordsmoen S, Birkenaes AB, et al. Medication adherence in outpatients with severe mental disorders: relation between self-reports and serum level. J Clin Psychopharmacol. 2010;30(2):169-75.

150. de Boer IM, Prins JM, Sprangers MA, Nieuwkerk PT. Using different calculations of pharmacy refill adherence to predict virological failure among HIV-infected patients. J Acquir Immune Defic Syndr. 2010;55(5):635-40.

151. Ware NC, Wyatt MA, Tugenberg T. Social relationships, stigma and adherence to antiretroviral therapy for HIV/AIDS. AIDS Care. 2006;18(8):904-10.

152. Stevens PE, Hildebrandt E. Pill taking from the perspective of HIV-Infected women who are vulnerable to antiretroviral treatment failure. Qual Health Res. 2009;19(5):593-604.
153. Gruskin S, Tarantola D. Universal Access to HIV prevention, treatment and care: assessing the inclusion of human rights in international and national strategic plans. AIDS. 2008;22(Suppl 2): S123-32.

154. Franke MF, Murray MB, Munoz M, et al. Food insufficiency is a risk factor for suboptimal antiretroviral therapy adherence among HIV-infected adults in urban Peru. AIDS Behav. 2011; 15(7):1483-9.

155. Senkomago V, Guwatudde D, Breda M, Khoshnood K. Barriers to antiretroviral adherence in HIV-positive patients receiving free medication in Kayunga, Uganda. AIDS Care. 2011;23(10): 1246-53.

156. Serrano C, Laporte R, Ide M, et al. Family nutritional support improves survival, immune restoration and adherence in HIV patients receiving ART in developing country. Asia Pac J Clin Nutr. 2010;19(1):68-75.

157. Boyer S, Clerc I, Bonono CR, Marcellin F, Bile PC, Ventelou B. Non-adherence to antiretroviral treatment and unplanned treatment interruption among people living with HIV/AIDS in Cameroon: individual and healthcare supply-related factors. Soc Sci Med. 2011;72(8):1383-92.

158. Kisenyi RN, Muliira JK, Ayebare E. Religiosity and Adherence to antiretroviral therapy among patients attending a public hospital-based HIV/AIDS clinic in Uganda. J Relig Health. 2011. doi:10.1007/s10943-011-9473-9.

159. Airoldi M, Zaccarelli M, Bisi L, et al. One-pill once-a-day HAART: a simplification strategy that improves adherence and quality of life of HIV-infected subjects. Patient Prefer Adherence. 2010;4:115-25.

160. Bangsberg DR, Ragland K, Monk A, Deeks SG. A single tablet regimen is associated with higher adherence and viral suppression than multiple tablet regimens in $\mathrm{HIV}+$ homeless and marginally housed people. AIDS. 2010;24(18):2835-40.

161. Juday T, Gupta S, Grimm K, Wagner S, Kim E. Factors associated with complete adherence to HIV combination antiretroviral therapy. HIV Clinical Trials. 2011;12(2):71-8.

162. Nelson M, Girard PM, Demasi R, et al. Suboptimal adherence to darunavir/ritonavir has minimal effect on efficacy compared with lopinavir/ritonavir in treatment-naive, HIV-infected patients: 96 week ARTEMIS data. J Antimicrob Chemother. 2010;65(7):1505-9.

163. Bangsberg DR. Less than $95 \%$ adherence to nonnucleoside reverse-transcriptase inhibitor therapy can lead to viral suppression. Clin Infect Dis. 2006;43(7):939-41.

164. Parienti JJ, Bangsberg DR, Verdon R, Gardner EM. Better adherence with once-daily antiretroviral regimens: a metaanalysis. Clin Infect Dis. 2009;48(4):484-8.

165. d'Ettorre G, Forcina G, Ceccarelli G, et al. Adherence and genotypic drug resistance mutations in HIV-1-infected patients failing current antiretroviral therapy. J Chemother. 2011;23(1): 24-7. 